\title{
Status of Global Energy Confinement Studies
}

\author{
S.M. Kaye; Cris W. Barnes; M.G. Bell; J.C. DeBoo; \\ M. Greenwald ${ }^{\S}$ K. Riedel, D. Sigmar
}

\section{Abstract}

Empirical scaling expressions, reflecting the parametric dependence of the L-mode energ: confinetnent time, have been used not only as benchmarks for tokamak o: eration and theories of energy transport, but for predicting the performance of prof ed tokamak devices. Sevesal scaling expressions based on data from small- and mediumsized devices have done well in predicting performance in larger devices, although great uncertainty exists in extrapolating yet farther. into the ignition regime. Several approaches exist for developing higher confidence scaling expressions. These include reducing the statistical uncertainty by identifying and filling in gaps in the present database, making use of more sophisticated statistical techniques, and developing scalings for confinement regimes within which future devices will operate. C'onfidence in the scaling expressions will be increased still if the expressions can be more directly tied to transport physics theory. This can be done through the use of dimensionless paramelers, better describing the edge and core confinement regimes separately, and by incorporating transport models directly into the scaling expressions.

- Princeton Plasma Physics Laboratory, Princeton Unisersity, Princeton, N.J. 08543

'Los Alamos National Laboratory, Los Alamos, N.M. 87545 and PPPL, Princeton, N.J. 08543

tGeneral Alomics, San Diego, Cal. 92138

${ }^{5}$ Plasma Pusion Center, Massachusetts Institute of Technolog:, Cambridge. Mass. 02139

Courant Institute, NYL, New York, NY 10012

Oak Ridge National Laboratory, Oak Ridge, Tenn. 37831 


\section{Introduction}

The global energy confinernent time, $\tau_{E}$ (defined as stored energy/power input), is one of the most direct and routine measurements made in tokamak plasma discharges. Its measured value depends on fewer assumptionss or calculations than any other transport quantities such as heat flux or heat diffusivity. This is because the total stored energy and input power can be measured nearly directly with high accuracy in almost every discharge. Furthermore, since similar measurement techniques are used by almost all tokamak experiments, the values of $\tau_{E}$ are straightforward to compare. Consequently, $\tau_{E}$ has been, and will always remain, the single most important absertable of tokamak confinement.

Global scaling expressions (GSEs) based on $\tau_{E}$ as a figure of merit serve to characterize large quantities of similar information from different experiments for very useful purposes. As a compact summary of data trends, often aigebraic and easy to remember, GSEs provide a characterization of tokamak confinement behavior that can guide both experimentalists and theorists. For experimentalists, GSEs are valuable, and thri are used as benchmarks for comparing different types of tokamak operation. Such comparisons help uncover new trends and new regimes, and they provide criteria for identifying regimes of "enhanced" confinement operation that need to be explored in more detail. For theory, scalings act as a set of guidelines that theories should try to simulate, or at the very least. not be inconsistent with. Even more, the trends of the GSEs can help motivate which models should be explored by providing a glimpse into the types of physics that may be important. Density dependence in the linear ohmic confinement regime, plasma current dependence in auxiliary heated regimes. length dependence, and isotope dependence all serve to constrain the classes of theories that may be determining the local transport if! tokamaks. ${ }^{1 \rightarrow}$

It is to be stressed that. at present. the most important and justifiable uses of GSEs are as descriptors. Exact predictions for future machines, based on GSEs, are highly suspect since the predirtions are usually in dimensional parameter regimes that lay outside the rarge on which the GSEs are based. Nunetheless, as will be seen, : 
scveral scaling expressions that were based on mid- and smali-sized devices have done a good job in predicting the performance of much larger size devices.

In Section II of this report, we wi]l discuss the status of GSEs. how the scalings were developed, the sources of major uncertainty in the scalings, and the limitations in the descriptive capabilities of the scalings. Section III will deal with how advanced regression techniques can be used to better determine scalings, and the need for public domain data and which data to use. Section IV will outline formalisms that will lead to better connection between GSEs and theory. It is this better connnection to theory that can raise the confidence leve] for using GSF, to predict performance in future devices.

\section{Status of Scaling Expressions}

\section{A. "Original" Scaling Expressions}

Global scaling expressions have historically been used both to benchmark tokamak performance and to guide the theoretical interpretation of energy transport. Here, we will briefly discuss some of the empirical scalings based on ohmic experiments, but we will focus on scalings derjved from auxiliary heating experiments, as thesc are deemed most relevant to reactor scenarios.

Most global scaling expressions are cast in terms of a poser law relation. The assumption of this parametric form is made primarily to cause of its convenicsce and simplicity coupled with a good characterization of dzta trends. The derivation of a power law expression rests un an assumption that there is a linear relation between the logarithm of the energy confinement time and the logarithms of a set of plasma parameters that are independent, so that

$$
\vec{Y}=\vec{B} \vec{X} \cdot \vec{C}
$$

where $\vec{F}=\log \left(\tau_{E}\right), \vec{H}$ is a vector containing the lugarithrns of the plasma (regressor) variables, $\vec{\epsilon}$ is the Gaussian distributed random error, and $\vec{\beta}$ is the least squares estimator, the vector containing the regressions coefficients. which is determined by 
minimizing the sum of squares

$$
\min \sum_{i} Y_{i}-\beta_{i} X_{i}{ }^{2}
$$

The major implicit assumptions made in this treatment are that

1. the linear model is correct and only a finite number of free parameters must be determined

2. no other physical variables other than the ones chosen for the regression have a significant impact on energy confinement (e.g., those related to edge or scrapeoff plasma)

3. the regressor variables are measured without error

4. there is no statistical difference among scalings for each individual tokamak.

One of the earliest characterizations of confinement trends across several experiments was the Alcator scaling, developed by Jassby, Cohn, and Parker, ${ }^{5}$ where $\tau_{E}$ was found to be $\propto \bar{n}_{\mathrm{z}} a^{2} q^{1 / 2}$. The ohmic study of Hugill and Sheffeld ${ }^{6}$ indicated $\tau_{E} \propto \bar{n}_{e}{ }^{0.65} M_{e j f}^{0.8} a^{1.6} B_{t}^{0.8}$ for a four parameter fit. Modifications to the Alcator scaling were made by Pfejffer and Waltz, ${ }^{7}$ where they found that $\tau_{E} \propto \bar{n}_{E} a R^{2}$, and the MIT group $^{8}$ with $\tau_{E} \propto \bar{n}_{e} a^{1.04} R^{2.04}$. Goldston ${ }^{9}$ later proposed the neo-Alcator scaling be combined with the $q^{1 / 2}$ scaling found in the carlier studies; there is still no consensus on the q-scaling in ohmic discharges for this nonsaturated regime.

The early work with neutral beam itjection indicated confinement trends whose global parametric dependences were quite different than those observed in ohmic plasmas. Little or me density dependence was ubserved, although $\tau_{E}$ was found to increase nearly linearly with plasma curtent and degrade as $P_{h \text { hal }}^{-1 / ?}$. Although there is not the same explicit dependence of $\tau_{E}$ on $I_{p}$ and $P_{\text {heat }}$ in ohmic and auxiliary heated plasmas, such a dependence for ohmic discharges cannot be ruled out because of the implicit dependence of $P_{\text {heat }}$ on $I_{p}$.

A description of the most commonly used dimensional scaling expressions that describe neatral-beam-heated discharge confinement through 1985 is given in the review 
article by Kave. ${ }^{10}$ As described in the article, these scaling expressions were based on data measured by magnetic flux loops from mid- and small-sized devices (e.g., DIII, PDX, ASDEX, ISX-B, and DITE), these data making up what was to be known as the L-mode database. At that time, the $\mathrm{H}$-mode and other enhanced confinement regimes (i.e., "Z-mode" of ISX-B) had been discovered, but the "unenhanced" confinement regimes were still the ones best documented.

The Goldston ${ }^{9}$ scaling combined an ohmic and auxiliary heating term in inverse quadrature fashion to form a global $\tau_{\varepsilon}$;

$$
\left(\frac{1}{\tau_{E}}\right)^{2}=\left(\frac{1}{\tau_{E}^{O H}}\right)^{2}+\left(\frac{1}{\tau_{E}^{\text {aut }}}\right)^{2}
$$

The ohmic confinement time,

$$
\tau_{E}^{O H}=10.2 \bar{n}_{e} a^{1.04} R^{2.04} q^{050}
$$

was of a form similar to that developed by Pfeiffer and Waltz ${ }^{7}$ and the MIT group. ${ }^{\mathrm{B}}$ The auxiliary term,

$$
\tau_{E}^{a u v}=30.2 M_{e f f}^{0.5} \kappa^{0.5} I_{p} P_{\text {beal }}^{-0.5} a^{-0.37} R^{1.75}
$$

exhibits a nonsize variable dependence which was a rough average of the parametric dependences reported by four devices (ISX-B, PDX, DIll, and ASDEX), and the size variable scaling derived from differences in confinement times amung the devices for a given set of operating conditions. The mass dependence was added later to reflect the isotope effect as observed on various experiments. In the above, and for all the expressions following, the units are: $\tau_{E}$ in msec, $l_{p}$ in $11 \mathrm{~A}, B_{t}$ in $\mathrm{T}, n_{\varepsilon}$ in $10^{19} \mathrm{~m}^{-3}$, $a$ and $R$ in $\mathrm{m}$, and $P_{\text {heat }}$ in MIV. For plasmas with neutral beam injection, $M_{e f f}$, the effective plasma mass in A.ML, is taken to be the average of the plasma and beam species; for RF only plasmas (see next section), $M_{\mathrm{c} f}$ is taken to be the majority species.

The Kaye-Goldston ${ }^{11}$ expression,

$$
\tau_{E}=24.6 M_{e f f}^{0.5} \kappa^{0.28} J_{p}^{1.24} n_{e}^{u .26} B_{i}^{009} P_{h+a 1}^{0.58} a{ }^{0.49} R^{1.65}
$$


in comparison. was based on a large number $(>600)$ of neutral-beam-heated discharges from various devices, and it was developed using a two-step multiple linear regression model. A modiffed form of the Kaye-Goldston expression, called "Neo-Kaye," was determined by excluding the DITE data, much of which turned out not to be in steady-state, and including early. TFTR NBI data. Buth this expression.

$$
\tau_{E}=44.7 M_{e f}^{0.5} \kappa^{0.28} I_{p}^{1.12} \bar{n}_{f}^{0.14} B_{i}^{0.04} P_{h e a l}^{p-0.59} a^{-0.04} R^{1.31}
$$

and the original Kaye-Goldston expression exhibited a stronger current dependence than did Goldston, but all three fit the then existing database comparably well.

One problem with the scaling expressions developed during that time period was the size. and more specifically, the aspect ratio scaling. The aspect ratio of the machines on which the scalings were based varied only between 2.5 and 4 , leading to a large uncertainty in the dependence on this parameter. Linfort unately, the uncertainty in the dependence on $R / a$ has not diminished even with the addition of data from the generation of larger tokamak experiments, whose aspect ratios are still within the range of 2.5 to 4 .

Other scaling expressions that have emerged over the course of the years include:

\section{Merezhkin-Mukhovatov ${ }^{12}$}

$$
\tau_{E}=12.0 M_{e f f}^{.5} \kappa^{.56} I_{p}^{-0.67} \bar{n}_{e} B_{t}^{.67} P_{\text {treat }}^{-.33} a^{2} R^{1.67}\left(\frac{R}{a}\right)^{-0.17}
$$

for discharges with

$$
\dot{n}_{e} \leq 153.33 B_{t}\left(\frac{B_{t}}{R}\right)^{0.33}\left(\frac{a}{R q}\right)^{1.67} .
$$

At otherwise fixed discharge conditions, the Merezhkin-Mukhovatov expression indicates an inverse dependence of $\tau_{E}$ on $l_{p}$, opposite to what is observed in $L$-mode discharges. However, in many cases. increases in $\tilde{n}_{e}$ and $B_{t}$ are coupled to increases in $I_{p}$ so that the positive dependence of $\tau_{E}$ on the first two parameters compensates for its inverse dependence on $J_{p}$. Consequently, in these experiments, the apparent dependence of $\tau_{E}$ on $J_{p}$ is in the correct sense. 
$\mathbf{T}-10^{13}$

$$
T_{E}=90.0 \text { a } R B_{f} \kappa^{0.5} P_{h e a l}^{-0.4}\left[Z_{e f f}^{2} I_{p}^{4} / a R q^{3} \kappa^{1.5}\right]^{0.08}
$$

There are two "offset-linear" scaling expressions:

Rebut-Lallia ${ }^{14,15}$

$$
\begin{gathered}
\tau_{E}=W_{\text {tot }} / P_{\text {heat }} \\
W_{\text {tot }}=2 W_{e} \\
W_{\text {tot }}=36.8 \mathrm{M}_{e f f}^{1 / 2} I_{p}^{1 / 2} \bar{n}_{e}^{3 / 4} B_{t}^{1 / 2} Z_{e f f}^{1 / 4} l^{11 / 4}+17.0 M_{e f f}^{1 / 2} I_{p} I^{3 / 2} P_{\text {heat }} Z_{e f}^{1 / 2}
\end{gathered}
$$

where

$$
l=\left(R a^{2} \kappa\right)^{l / 3}
$$

The coefficients of the Rebut-Lallia fit were adjusted to take intu account an $\mathrm{Weff}_{\mathrm{e}}$ correction not in the original formula. The original formula was based on $D^{\circ} \rightarrow$

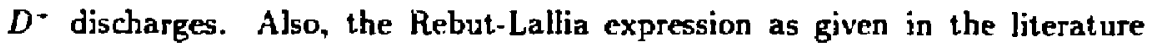
describes electron confinement only; here, the conservative assumption that the total stored energy is twice the electron stored energy, $W_{t o t}=2 W$, was made.

\section{Shimomura-Odajima ${ }^{16}$}

$$
\begin{aligned}
& \tau_{E}=\frac{I Y_{O H}}{P_{\text {heat }}}-\tau_{1 \mathrm{in}} \\
& H_{O H}=15.6 M_{e f f}^{1 / 2} \kappa^{0.2} I_{p} \bar{n}_{e}^{0.6} B_{t}^{0.2} a^{0.4} R^{1.6} f\left(Z_{e f}\right) g\left(q_{e y l}\right) \\
& \tau_{\text {ine }}=84.9 \kappa a^{2} M_{e f f}^{1 / 2} \\
& f\left(Z_{\mathrm{eff}}\right)=Z_{\mathrm{eff}}^{0.4}\left[\frac{\left(15-Z_{\mathrm{ef}}\right)}{20}\right]^{0.6} \\
& g\left(q_{c y l}\right)=:\left[3 q_{c y l}\left(q_{c y l}-5\right)\left|;\left(q_{c y l}-2\right)\left(q_{c y l}+7\right)\right|^{0.6}\right.
\end{aligned}
$$

Sote the lack of a plasma current dependence in the $\tau_{2 n c}$ term of the ShimomuraOdajima expression; this will be discussed more further on.

In all the scaling expressions given here, the plasma species dependence. $M_{e \theta}^{1 / 2}$, was incorporated a pusteriori, since must of the expressions were developed from a database with only one effective masa (i.e., in Goldston and Kaye-Goldston the plasma 
species was a mix between $H^{-}$and $D^{+}$). The species dependence was assumed to be $M_{\text {eff }}^{1 / 2}$, based on results from ASDEX, ${ }^{17}$ DIII- ${ }^{18}$ and JFT-2M. ${ }^{19}$ although it should be pointed out that no isotope dependence was found in $\mathrm{L}$-mode discharges in $\mathrm{JET}^{20}$ and DIII-D. ${ }^{21}$ Clearly, the mass dependence of confinement is still not well understood.

In addition to the outstanding questions regarding aspect ratio and species effects, the dependence on plasma elongation is also uncertain. The $\kappa^{2028}$ dependence in KayeGoldston, for instance, is derived from a $\kappa^{0}$ dependence in ISX.B discharges and a $\kappa^{0.46}$ dep-ndence in DIII discharges. It is not clear that an avrage of these coefficients. even weighted by their variances as is done in Kaye, ${ }^{11}$ is justified. The averaging essentially assumes that both $\kappa$ dependences are describable by the same parent distribution; in fact, the different behavior may be indicative of different physics between the machines, and if this is the case, such averaging is not appropriate. Additional information on this parametric dependence can come from experiments on DIJI-D and JET, machines that can vary elongation at otherwise fixed conditions.

\section{B. Recent Data and Scaling Expressions}

\section{Database}

In order to assess the goodness of fit of the original set of scaling expressions to the present generation of tukamak experiments, the original neutral beam heating database as described by Kaye and Goldston" was augmented by data from newer and larger devices, bringing the total number of L-mode discharges in the clatabase to approximately 1800 . Table I lists the tokamak experiments, number of L-mode discharges used from each experiment. and constraints on the selections of these discharges. Experiments with ICRF, in addition to neutsal beam heating, were performed on JET. JT-60. and JFT-2M. Lower Hybrid heating expcriments were also performed on JT-60. For the tokamaks with RF and neutral beam heating, no difference in either the absolute or relative confinement trends could be observed for various heating scenarios. Consequently, no distinction between XBI and/or RF heating was made for the purposes of this study. although it should be pointed out that the avail- 
able RF power was small compared to that for neutral beam heating. and in some cases was comparable only to the ohmic heating power.

The data received from each machine differed in terms of the number and types of parameters. A common subset of global parameters was selected for the primary database. These were:

$\begin{array}{ll}a & \text { plasma minor radius } \\ R & \text { plasma major radius } \\ \kappa & \text { elongation } \\ l_{p} & \text { plasma current } \\ B_{t} & \text { toroidal field } \\ n_{e} & \text { line-ateraged electron density } \\ \beta_{t} & \text { volume-averaged toroidal beta } \\ \beta_{p d} & \text { poloidal beta } \\ V_{i} & \text { lorp voltage } \\ P_{\text {heat }} & \text { total heating power (auxiljary }+ \text { ohmic) } \\ E_{\text {inj }} & \text { neutral beam injection energy } \\ q_{\text {shaf }} & \text { Shafranov q } \\ M_{e f j, b e a m} & \text { beam species } \\ M_{e f j, j l a s} & \text { plasma species } \\ \tau_{E} & \text { energy confinement time }\end{array}$

The plasma betas and confinement times were based on equilibrium .1HD values, which included the fast ion component. These were chosen wer the direct diamagnetically measured values to avoid any effects due to different pressure anisotrupies among the durices. PLT data were excluded for any hut comparison purposes, as this dataset was $n+1$ based on magnetics and was thus inconsistent with the remainder of the dataset. The data in the dataluase were obtained from the steady-state or near steady-state portion of the discharge. When possible. this was checked and the data typically satisfied the relation $d I I ; d t \leq 0.15-0.20 \%$ te:at As mentioned, the DITE data were excluded for most purposes since many of these discharges were not in either energy or density equilibrium. 
For most tokamaks with divertors, operation in that configuration led to $\mathrm{H}$-modes. In JT-60, divertor operation led to poorer confinement than in limiter discharges for the current rasge from 2.5 to $2.8 \mathrm{MA}$. JFT-2M data in both the L-and H-modes exhibited a great deal of scatter. Selected operating parameters for the discharges in the database are given in Table II.

\section{Aspect Ratio}

As mentioned earljer, the limited range of aspect ratio in the original database led to a large uncertainty in the scaling of $\tau_{E}$ with this parameter. This is unfortunate, since the true dependence of $\tau_{E}$ on aspect ratio may indicate the inportance of trapped particle modes. The data in the expanded database do little to correct this shortcoming. The range of currents and aspect ratios spanned by the various experiments is shown in Fig. 1. As can be seen in the figure, the range of aspect ratio is limited. The only two high aspect ratio experiments for which data have been obtained are DITE and TFR. DITE data were generally excluded from analysis since many of the discharges were not in equilibrium, and there were only four TFR points. Hence, the effective range of aspect ratio was only from 2.5 to 4 . A second feature of Fig. I is that the aspect ratio and current (or size) appear to be coupled; in general, the lowest aspect ratio experiments have the highest currents (or largest size). A dependence not seen explicitly in the figure is that between elongation and aspect ratio, in which, at least for the larger devices, the linest aspect ratio experiments are the ones that can produce plasmas with Jarge elongations. The existing interdependences among these parameters make it somewhat unjustified to treat them as truly independent variables.

There eyist experiments that can be performed and data comparisons that can be nade that can yield more information on the dependence of $\tau_{E}$ and aspect ratio. For one, J'BX-M operates at an aspect ratio of 5.5 (see Fig. 1) at high elongation. However, PBS $V$ data are not entirely compatible with the others since it is the only device to operate with indented plasmas.

There are experiments outside the nominal operating range that can yield the 
desired aspect ratio information. Results from TFTR operating at $\mathrm{R}=3.1 \mathrm{~m}, \mathrm{a}=0.44$ $\mathrm{m},(\mathrm{R} / \mathrm{a} \sim \mathrm{7}-8)$ and low power and current can be directly compared to those at the same minor radius from $P D X$ with $R \simeq 1.44(R / a \sim 3.3)$. In addition, data from DII $1-D$ $(R=1.65 \mathrm{~m}, a=0.65 \mathrm{~m}, \mathrm{R} / \mathrm{a}=2.5)$ can be compared to data at the same major radius from D-shaped or only slightIy indented plasmas on PBX-M $(\mathrm{R}=1.65 \mathrm{~m}, \mathrm{a} \leq 0.30 \mathrm{~m}$, $R / a \geq 5.5$ ). Some correction for operation at different currents may have to be made in this latter comparison. Size scaling at similar aspect ratio can be obtained by comparing data from DIII-D and JET. Therefore, while it appears that the neressary information can be obtained, it will require dedicated run time on several experiments.

\section{Scaling Expressions}

The development of "updated" scaling expressions based on the expanded database can follow two lines. The first is to simply take all the data in the database and run it through a simple multiple linear regression routine. The advantage of this approach is that it is simple; the disadvantage is that the resulting expression is biased by the trends contained in the dataset from the experiment with the greatest number of points and variation, which in this case is JET. Here, the assumption that the regressor variables are linearly independent is still made. The scaling emerging from such a treatment is:

$$
\tau_{E}=93.6 M_{e B}^{0.32} \kappa^{0.36} I_{p}^{0.86} \tilde{n}_{e}^{0.10} B_{t}^{0.25} P_{\text {heat }}^{-.54} a^{0.72} R^{0.49}
$$

with $R^{2}=0.93$, where $R^{2}$ is the coefficient of varjation of the fit. The fit was dereloped without including DITE and ISX-B data; these two machines are the smallest devices with significant numbers of points in the database. The experimental vs. fitted $\tau_{E} s$ are plotted in Fig. 2a.

To avoid the bias introduced by the simple approach, a more complex method, as perforned by Kaye and Goldstun ${ }^{11}$ was used. In this approach, the parametric dependence of $\tau_{E}$ with nonsize variables is determined for each size-constrained dataset. The parametric dependences for each machine are given in Table III.

What is of interest in the table is the less than linear rurrent depcndence in the 
bigger devices operating at higher powers and currents. It is not known whether there is an implicit dependence of the $J_{p}$ regression coefficient on current, power, size, or some dimensionless variable. However, the results shown in the table point out dist inct differences between the groups of the large, and mid- and small-sized devices, with the larger devices exhibiting a weaker $l_{p}$ dependence. This feature underscores the real possibility that the differences in scaling among the individual devices may be real and significant, and they may indicate true physics differences among the machines. Consequently, developing an "average" scaling would be justified only in the most global sense in order to characterize the physics that is common among the various experiments. What is also needed, however, is to understand the physics basis for differences in the various machine trends.

Despite possible machine-to-machine differences, it is still useful to construct average scalings in order to define grossly the confinement behavior across a full range of tokamaks. Following the method of Kaye and Goldston, and using data from all of the machines given in Table III, the following scaling is obtained:

$$
\tau_{E}=52.1 M_{e f f}^{1 / 2} \kappa^{1 / 4} I_{p}^{0.85} \bar{n}_{e}^{0.10} B_{l}^{0.3} P_{\text {heat }}^{-0.5} a^{0.3} R^{0.85}
$$

with $R^{2}=0.91$. If data from only big machines (TFTR, JET, JT-60, DIlI-D) are used, the following scaling is obtained:

$$
\tau_{E}=52.1 M_{s f}^{1 / 2} \kappa^{1 / 4} I_{p}^{0.85} \bar{n}_{e}^{0.10} B_{t}^{0.3} P_{\text {heat }}^{-0.5} a^{0.8} R^{0.5}
$$

with $R^{2}=0.91$. These two scalings are shown in Figs. $2 b, c$. What is clear is that the scalings (10)-(12) are essentially the same; only slight differences in the regression coeffeients of the nonsize parameters are observed. It is important to note that the current dependence is slightly less than linear, in contrast to the pre-existing power law expressions (2)-(4). This is a consequence of the less than linear current dependence in the larger devices as discussed earlier. In addition, the newer scalings have less of an aspect ratio dependence (at constant $q$ and $B_{t}$ ) than the earlier ones (2)-(4), although, again, the uncertainty here is large. It is also interesting to point out that the combined exponents of the a and $R$ scaling yicld a true length scaling 
between $L^{1.15}$ and $L^{1.30}$ in (10)-(12), which is in the same range of length scalings exhibited by the early expressions (2)-(4).

\section{Statistical Comparison with Data}

It can be seen in Figs. 2a-c that while most of the discharge confinement times can be well represented by the scaling expressions, there remains a great deal of scatter

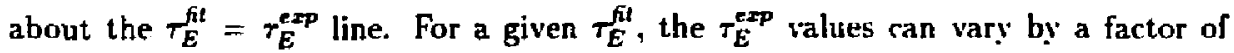
two. The scatter is caused by both pure experimental scatter due to systematic and random diagnostic errors, by changing and unquantifiable discharge conditions on a discharge-to-discharge basis, and by inherent errors due to the underlying assumption about the parametric form of the scaling expression to be used.

For the scaling expressions shown in the figure, the cuefficient of variation, $R^{2}$, is greater than 90\%. All of the scaling expressions except for Merezhkin-Mukhovatov, also exhibit. $R^{2} \geq 0.90$. This does not necessarily indicate that all the scaling expressions fit the data equally well, for $R^{2}$, which gives the percentage of variation in the dataset explained by the fit, is weighted strongly by the range of $\tau_{E}$ (e.g., $\left.1-10^{3} \mathrm{msec}\right)$ rather than the variation about the fit. Consequently, $R^{2} \rightarrow 1$ wheneser the range of $\tau_{E}$ is much greater than the variation about the fit, which in this case is by two to three orders of magnitude.

A more appropriate way to assess the goodness-of-fit of the various scaling expressions is to examine data from a series of single parameter srans at otherwise fixed discharge conditions. This assessment restricted itself to power scans at fixed current, major and minor radii, and where possible, density, toroidal field, and $Z_{\text {eff }}$. Power scans at different currents were examined for each of the newer machines (DIII-D, JET. TFTR, and J'T-60).

The results of this assessment clearly indicated dramatic differences in the goodnessof-fit among the various scaling expressions. While we will not go through all the detailed comparisons here, the comparison with JET will be shown. In. Fig. 3(a)-(j) are shown JET power scans ( 0 to $20 \mathrm{MW}$ ) taken at $1,3.5 \mathrm{MA} . \hat{n}_{\mathrm{e}}=1.5 .3,4 \times 10^{19} \mathrm{~m}^{-3}$, 
$B_{t}=2.1,2.7,3.5 \mathrm{~T}, Z_{e f}=4,3,2.5$, respectively, and $M_{e f f}=2, a=1.2 \mathrm{~m}, R=2.9 \mathrm{~m}$ and $\kappa=1.4$. Overlain on the sets of datapoints are the confinement time predictions (solid lines) for each scaling expressions for each individual scan. Reasonably good agreement with the data is found for Kaye-Goldston, Neo-Kaye, and Goldston, although the current scaling is slightly too strong in the first two. In these comparisons, the scaling expressions were not combined with the ohmic confinement in inverse quadrature fashion. Rebut-Lallia overpredicts the confinement times and exhibits tow strong a current dependence, Shimomura-Odajima agrees with the 5 MA points at low power, but it exhibits too weak a power degradation as well as too weak a current dependence (leading to a severe overprediction at low $I_{p}$ ). Merezhkin-Mukhovatov yields too weak an $I_{p} / \bar{n}_{e}$ dependence, underpredicting all but the lowest currrent points. Since $\bar{n}_{e}$ and $B_{t}$ both increase with $I_{p}$ in these JET scans, the MerezhkinMukhovatov scaling in this case exhibits the correct apparent current scaling. The T-10 scaling exhibits ton weak a current depender e; here, a mumerical coefficient of 40 instead of 90 (Eq. 6) was used, as the coefficient of 40 is the best fit normalization to the L-mode database. Of the three new scalings, "Big" (12) fits the data the best, with the "All" (10)-(11) scalings underestimating $\tau_{E}$ by $\sim 15 \%$ at the higher currents. While the details of the fits are somewhat different for the other three devices, the JET comparison reflects the overall conclusions concerning goodness-of-fit of the various scalings.

In order to represent adequately the goodness-of-fit of the various scalings in a statistical sense, the varjable that represents the variation of the data about the fit was used. This variable known as the "Root Mean Square Prediction Error," is given hy:

$$
\text { R.MSPE }=\left[\frac{1}{N} \sum_{i=1}^{N}\left(Y_{i}-Y_{f t, t}\right)^{2}\right]^{\frac{1}{2}}
$$

and satisfies the above requirement. ${ }^{22}$ The results of using this variable are shown in Fig. 4, and they tend to reflect the conclusions obtained by the detailed parameter scan comparisons. The power law scalings (1)-(4) and (10) $\cdot(12)$ describe the data the best, while the offset-linear scalings, T.10, and Merezhkin-.Mukhovatov do not do 
as well.

As previously pointed ont, the $\tau_{\text {inc }}$ term in Shimomura-Odajima scaling lacks any sort of a current dependence, the claim being that all the current dependence of the total $\tau_{E}$ is derived from that in the $W_{O H}$ term. However, the data in the database indicate otherwise. In Fig. 5, $\tau_{\text {ine }}$ is plotted vs. plasma current for a variety of tokamaks. Note that the PDX and DIII incremental confinement time and current values are multiplied by ten to allow them to fit on the same scale. What is clear from the figure is that all the machines exhibit a dependence of $\tau_{2 n c}$ on $I_{p \text {, although not }}$ necessarily linear. It is most likely the case that the Shimumura-Odajima scaling is more appropriate in the $q \leq 3$ range, where the dependence of confinement on plasma current disappears; this effect was first observed on DIII by the JAERI ${ }^{23}$ group, but first recognized as a $q \leq 3$ effect by the DIII-D group. ${ }^{2 !}$

\section{Additional information needed for scaling studies in regimes of enhanced confinement}

The L-mode scaling expressions developed over the last decade (Goldston, KayeGoldston, etc.) provide a reasonable description of the lower limit to the confinement time observed in many tokamaks. As discussed, all of the large tokamaks produce energy confinement times dusing high-power auxiliary heating which are close to and scale with the L-mode value when running plasmas with unconditioned limiters and/or strong gas puffing. For this reason, most experimenters have described regines of improved confinement in terms of the enhancement factor of the entrgy confinement time over the predictions of L-mode scaling. In the absence of scaling expressions derived specifically for each of the regimes of mhanced confinement, the tendency has then grown to use L-more scaling multiplied by a constant enhancement factor to predict the performance of the enhanced confinement regime in another device. For examnle, the prediction of the confinement time for the $\mathrm{H}$-mode is often taken to be a factur. usually about 2 , times the $\mathrm{L}$-mode scaling value far particular conditions of plasma dimensions, plasma current. and heating power. The uncertainty inherent in 
this approach is sometimes acknowledged by allowing the enhancement factor to vary over a range. This situation is unsatisfactory, especially since some of the enhanced confinement regimes scale differently with the basic $L$-mode variables and depend also on parameters not present in the L-mode expressions. Additionally, most enhanced confinement discharges are not in steady-state; consequently, the enhancement factor may indicate only the maximum confinement achieved, irrespective of its duration.

A plethora of supposedly distinct regimes of enhanced confinement has been described in the tokannak literature in the last decade, including the H-mode. ${ }^{23-29}$ the Z-mode (confinement improvement perhaps due to impurity-produced peaked density profiles), ${ }^{30}$ the enhancement of confinement by hydrogen pellet injection, ${ }^{31-36}$ the supershot regime, ${ }^{37}$ and more recently the Improved Ohmic Confinement (IOC) of ASDEX $^{38}$ and the Improved Divertor Confinement seen in JT-60. ${ }^{39}$ Each of these regimes may exhibit its own peculiar scaling characteristics and limitations. The most obvious diffculty in developing a scaling for an enhanced confinement regime is to decide whether particular discharges belong to that regime of confinement so that data san be compared from machine to machine. Even the most universal of these :egimes, the $\mathrm{II}$-mode, is manifested somewhat differently on different tokamaks. For example, in DIII, distinct transitions to the H-mode were not observed whereas in DIII-D, they are. The behavior of impurities during the $\mathrm{H}$-mode and the effect of the position of the $\mathrm{X}$-point in relation to the $\nabla B$-drift direction on the $H$-mode threshold vary. Furthermore, the observation of $\mathrm{H}$-Inode characteristics in limiter plasmas ${ }^{29,40,47}$ complicates the classification of $\mathrm{n}$-mode phenuliena.

Another difficulty is that even in well-defined regimes of enhanced confinement, the quality of confinement is quite variable; e.g., instances of $\mathrm{H}$-mode discharges without any confinement enhancement have been descrihed in certain cunditions. If global scaling expressions are to be of value in the operation of present and the design of future experiments, they must reflect the dependencies of confinement upun all the plasma parameters over which we bave control. Unfortunately, it appears that many of the experimental conditions which have been identified as critical for the enhancement of confinement in a particular regime are not easily expressible as parameters 
which can appear explicitly in a scaling expression. Furthermore, conditions which are critical for one regime of enhanced cunfinement may not be applicable to any other regime. Clearly, the solution to this problem wonld be to cast general scaling expressions in terms of the underlying, correctly dimensioned plasma parameters which control transport and then to relate these plasma parameters, in a physically understandable way, to machine variables. The dependence of confinement time on peaking of the density profile observed in Alcator-C, TFTR, and ASDEX provides an example of the use of such a plasma parameter (e.g., $n_{r}(0) / n_{e}$ in describing confinement scaling). In TFTR supershots, the tendency for density peaking to increase with neutral beam injection power and to decrease with plasma current then serves to explain the differences between the power and current scalings of supershots and L-mode discharges, although the exact relationship of the peaking factor to the external conditions, particularly the plasma current, is not well understood. This reflects the fact that, in a system as complicated as a tokamak, it is unlikely that general scaljing expressions can be found in such ideal physical terms. It is probable that scaling expressions in terms of machine parameters (e.g., plasma dimnensions, plasma current, toroidal field, input power) supplemented by simple profile characterization data and device-specific information (e.g., boundary clearances of target plasma density) will continue to be used. Thus, in a general database of enhanced confinement studies, there will inevitably be a great deal of missing data and some superfluous data, representing the differences from regime to regime in relevant variables.

\section{Progress Towards a Better Empirical Understanding}

While the ultimate goal of tokamak transport studies is to understand the physics that regulates plasma confinement, the empirical approach. in the interim, has its utility in guiding both theory and experiment. We have seen that the first generation of GSEs used rather basic regression techniques along with data from a narrow range of experiments. Thus, the GSEs themselves had limitations in their validity and confidence levels. In this section, we shall explore two essential issues for defining 
the confinement trends with more certainty than in the past, these being the use of advanced regression techniques and expanding the type of data in the database. The discussion of the former topic will serve merely as a summary of some of the detailed work by Riedel. ${ }^{42}$ In the discussion of the latter topic we will nuake recommendations on how to expedite placing data in the public domain.

\section{A. Advanced Regression Techniques}

One of the major flaws in the previous analysis of tokamak data was the assumption that the scaling variables were truly independent from one another. The fact that this colinearity among the varjables exists means that not all the variables have been varied independently, and that some can be eliminated from the scaling expressions. It was shown earlier that two of the important scaling variables, $r$ and $R / a$, were not entirely independent within the dataset used. In addition, in some experiments such as JET, plasma current, toroidal field, and line-averaged plasma density were coupled. This interdependence naturally leads to poorly determined regression coefficients. Several approaches to ameliorate this situation can he taken. First of all, experiments can be designed to obtain the necessary information. Secondly, techniques to reduce colinearity of the regressor varjables are available. One such techrique that may help in this regard is Principal Component Analysis (PCA). PCA is as eigenvector-eigenvalue solution that yields each eigenvector (combination of scaling variables) and its variation (magnitude of the eigenvalue). In this analysis, the eigentector with the smallest eigenvalue is the direction of least variation. and experiments should be planned to expand the variation in this direction. In the case of global scaling, this eigenvect of is $\kappa^{0.4}(R / a)^{0.9}$, which means that high/low elongation at high/low aspect ratio experiments are needed. Another approach to addressing pororly determined regression coefficients is to employ a technique called Ridge Regression (RR). This is accomplished by introducing a small biased error into the analysis; the result of this is to reduce all of the regression coefficients by an amount proportional to the uncertainty in that component. In this way, Ridge 
Regression has an advantage over PCA in that RR reduces the importance of pnor]y conditioned eigenvectors while PCA merely identifies them.

In any regression analysis there will be a certain number of points that appear to the user as exceptions to the trends reflected by the majority of points. Typically, these points constjtute a much larger tail of "unlikely" events than would be expected for a Gaussian distribution. These statistical outliers can have a large influence on the determination of the fit coefficients since the points are weighted by their least squares error. Robust statistics seeks to minimize the influence of these outliers on the fit. However, statisticaj outliers should not simply be downweighted or discarded; often, these outliers indicated new physics regimes and a direction in which to push experiments. More may be learned about what will improve tokamak performance by studying outliers than by studying the "well-behaved" discharges.

Another issue in the advanced regression topic has to do with error estimates and predictive uncertair.ty. In the simple approach to linear regression, the assumption that all errors vary randomly from discharge-to-discharge was made. However, mary systematic errors are quite reproducible for a single tokamak from dischargetu-discharge. Botb the systematic and the random errors should be included in the least squares estimator; failure to do so leads to overestimates of the confidence in the scaling. Resampling techniques, such as jackknifing and bootstrapping, form a set of statistical methods from which model variance can be related to data variance without having to make any assumptions about the statistical propertics of the data. Use of these techniques can lead to better inodel estimates and standard errors. ${ }^{\text {.ta }}$

In order to make use of sorne of the regression techniques described above, a large number of regression diagnostics make it possible to understand better which datapoints have a large role in determining the regression and how these points may influence the fit coefficients. To assess grodness of fit, it is useful to plot the resjdual error $\epsilon_{i}$ versus the predicted value, $\dot{\gamma}_{i}$, as well as the individual independent variables. The residual plots aid in

1. locating the outlying datapoints where the fit is bad. 
2. finding clusters of residual errors, indicative of a correlated error structure,

3. identifying a heteroscedastic error structure (where the error variance is not constant over all variables),

4. identifying possible higher ordes terms that should be included as regressor variables, and

5. identifying terms which have no significant impact on the goodness of fit, except. possibly to fit outlying points.

To determine the influence of various groups of data on fits, one or more datapoints can be deleted and the fit recalculated. Thus, both the set of datapoints that significantly affect the fit and the particular parametric dependences can be isolated. Finally. cluster and discriminant analysis can be used to group data into discrete subsets which have different scalings, and to determine boundaries between groups of points, respectively.

\section{B. Database}

There has been a long standing need for publically accessible tokamak data, particularly with respect to transport and confinement. While such a database has existed, ${ }^{11}$ its contents have been restricted to global discharge parameters for L-mode discharges. Only recently has some enhanced confinement and profile data been incorpurated. There exist two issues for the collection and use of data: the protocol for using the data, and the definition of which data are to be collected. The technical problems of how to implement, store, and then distribute data in a national or international database are not as severe as establishing protocols on how the data are to be used and accredited, on when data should no longer be proprietary, and on what standards should be for the level of analysis.

The archival record for most tokamak experiments has traditionally been IAEA and conference reports as well as papers published in journals such as Nuclear Fusion. However, the space constraints of this form of data presentation most often do not 
allow complete reporting of the parameters needed for transport studies. This is particularly the case if one wishes to analyze the data in a novel fashion. Most of the information needed for these studies is stored at the site of the experiments on computer disks and tapes which are not readily accessible to the interested user. The global databases that have recently been in use do not contain a complete enough description of the plasma discharge for even a simplistic transport study.

The first problem to solve is how to make the data accessible with minimum restriction to a large number of users. We recommend that an informal data group be formed; this group would consist of one or two scientists handling the data collection and redistribution, and one representative from each contributing tokamak. Here we stress the word informal, since experience has shoun that the more informal a structure is the more efficient it tends to be. The role of the Data Group would be to ensure the availability and placement of data into the database.

The data that goes in should be analyzed data that form the basis of publications or invited papers (IAEA, APS, EPS, journal articles), which would set some standard of analysis, and should be incorporated into the database shorty after publication. The data would then carry a published record, even though all the data details will not likely have appeared in print. The users of the database would be required to cile the original publications as relevant, or some as yet to be established, more global citation referencing the state and contents of the database at specific times. To be useful, the database should truly be public; there should be no conditions an its use or interpretation other than paying heed to the caveats on the data provided by the contributors and acknowledgment of the data source.

Now we turn to the contents of the database. Existing L-mode datahases contain global data only, as discussed earlier. While these obvious global parameters form a basis for characterizing discharges, a more complete study of tokamak transport requires some profile information. A first stage to incorporating profile information would be including $T_{e}(0) /\left\langle T_{e}\right\rangle, n_{c}(0) /\left(n_{e}\right\rangle, P_{\text {rad }}(0) / P_{\text {rad }}^{\text {tot }}, Z_{e f f}$, and an estimate of heating profile peakedness for both NBI and RF scenarios, such as is given in Sugihara and Singer. ${ }^{44}$ Comments on the MHD activity at the time of interest would also serve 
to better characterize the data. This first order profile information will inevitably improve the state of L-mode databases enormously.

It is likely that full profile information will be available for only a small set of the above "Global+" database. The reason for this is that if any detailed profile information is to b- useful, practically all of it is required. Having detailed electron and ion temperature and density profiles available is of limited utility unless the detailed heating deposition profile is also available. It is also important to have the profiles of the fast particle population and energy content to be able to distinguish between the thermal and fast particle contributions. In some cases it may be important to know the calculated particle sources and sinks. In shaped plasmas further complications arise, as the profile data must be reported as a function of some magnetic flux, with the shape parameters specified at each surface. In short, enough profite dala should be available to carry out a standard transport analysis. The developmert of a database with this collection of detailed profile information should be encouraged.

The characterization of enhanced confinement regime data is even more problematic since, as discussed in the last section, there are additional parameters that are needed to describe the discharge confinement. In supershots, for instance, the quality of confinement depends primarily on two parameters, the density profile peakedness and the ratio of co- to counter-injected power. This assumes that the proper edge recycling conditions are met to obtain the peaked deusity profile. These two primary scaling variables are not included in L-mode GSEs, but the densit: profile peakedness may, in fact, be a crucial, unifying parameter for L-mode data as well. ${ }^{45}$ and may serve to link the supershot to the L-mode regime, with the current and power dependence in the L-mode perhaps being a reflection of the density peakedness dependence.

The quality of confinement in H-mode plasmas at constant "global" discharge parameters may vary up to a factor of two depending on many conditions. These include recycling conditions (divertor to main chamber compression ratio or main chamber neutral pressure), separatrix height from the divertor plates, wall cleaniness, plasmawall gaps, open vs. closed divertor, and frequency and magnitude of ElsMs. One of two approaches to characterizing $\mathbf{H}$-mode confinement is necessary. The first is to de- 
cide how to quantify all these effects and then incorporate them as scaling variables. The second, and more practical, is to restrict the data being analyzed for trends by certain criteria clearly delineating the discharge conditions (i.e., grassy ELA1s, $\geq 10: 1$ compression ratio, etc.). This second approach will require many separate analyses to characterize fully the confinement trends, but it has the advantage over the first in that at least some results will be obtained in a timely fashion.

\section{Progress Towards a Better Theoretical Understanding}

Up to now, the approach to developing global scalings, and the scatter in the data jtself, has been such as to prevent a definitive test of theoretical transport models. For instance, it was shown by Kaye ${ }^{10}$ that two global scalings. one based on a collisionless drift wave model and one based on high-n ballooning, brith described the data trends equally well. Additionally, both theories were compatible with the Kaye-Goldston or Goldston scalings. It is not surprising that no single theory is clearly superior since the plasma naturally divides itself into different physics regimns, the inner regirn dominated by sawteeth, the core region governed by "collisionless" transport, and the edge region regulated by transport driven by more resistive or collisional phenomeı. It is difficult to see how all three regimes can effectively be combined into one simple power law scaling with only a limited number of scaling variables. In this section, we will focus on means to address these theoretical issues within the framework of global scaling. In addition, we will touch on a topic that has received only limited attention. that is, the relation between energy and particle confinement. The objertive in this section is to show how GSEs cail be better connected to theory, for the more we include physics considerations in the GSEs, the more we improve their predictive capability and usefulness.

\section{A. Relation to Theory}

One of the more straightforward ways to connect global scaling better to theory is to base the -caling expressions on the dimensionless variables that are thought to 
reflect the physical transport processes. There can be no doubt that confinement varies with plasma current, heating power, etc., but it is difficult to relate these engineering variables to physical theory. A better approach would be, for instance, to use the Connor-Taylor constrained dimensionless variables ${ }^{46}$ such as $\beta, \nu^{\circ}$, and $\rho_{i} / a$, augmented by other dimensionless parameters such as $q_{m h d}, R / a, \kappa$, etc., as the basis for describing confinement trends. The coupling of current to size seen in recent experiments can be considered to be a constraint on $q_{m h d}$, for instance. It is also the case that there is less of an extrapolation to reactor-size devices with these dimensionless variables than with the standard dimensional ones. Regression analyses of $\tau_{E}$ on the above-mentioned variables would be one approach to understanding how confinement varies with these parameters, but this approach should be verified by controlled experimental scans using these dimensionless parameters as the scan variables.

To better understand tokamak thermal transport, the use of a confinement time definition that better represents the thermal energy is needed. The $\tau_{E}$ definition that has been used for global scaling studies up to now has been the global value; a value that contains fast ion, as well as thermal, energy. Additionally, the heating power value in the denominator can be an overestimate of the true heating power, for it does not take into account fast ion losses due to charge-exchange and bad orbits.

A better descriptor for $\tau_{E}$ would, of course, come from full radial profile information of the thermal and fast ion species. In the absence of this information, certain adjustments to the $T_{E}$ can be made in order to better characterize the transport loss. The first step is to subtract the fast ion component from the total energy 11 to to give only the energy in the thermal plasma, $W_{t h}$. While this fast ion contribution is not always calculated explicitly, for $\mathrm{NBI}$ it can be approximated by the value

$$
W_{\text {fast }}=\eta_{\eta} P_{\text {inj }} T_{\text {therm }}
$$

where $\eta$ is the power fraction of the full energy component of the beam (which can be set equal to one for these purposes), $P_{i n j}$ is the injected power, and $\tau_{t \text { therm }}$ is the 
beam thermalization time, given by

$$
\tau_{\text {eherm }}=\frac{\tau_{\text {dd }}}{3} \ln \left[1+\left(\frac{E_{\text {inj }}}{E_{\text {eril }}}\right)^{1.5}\right],
$$

where $\tau_{\text {sd }}$ is the slowing down time, $E_{i n j}$ the full injection energy, and $E_{r \text { rit }}$ the critical energy ${ }^{47}$ Once the fast ion component estimated in this fashion is subtracted out, the heating power can be modified by subtracting the total radiated power;

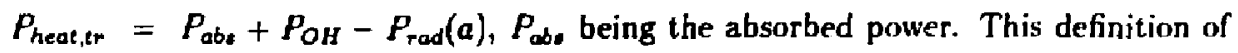
$P_{\text {heat,tr }}$ is still not totally representative since the $P_{\text {heat, }}$ does not acciunt for the charge-exchange and bad orbit loss, nor does it take into account the profile effects of $P_{\text {radi }} P_{\text {rad }}$ is typically small for $r \leq 2 a / 3$ and can dominate all loss channels for $r \geq 2 a / 3$. Other atomic loss processes of the thermal plasma are relatively small. Nevertheless, within the rnmsi raints rof glohal sraling, therefore, the hect indirator of the confinement resulting from turbulent transport processes is

$$
\tau_{E}^{t h}=W_{t h} / P_{\text {heat, }, \text { r }}
$$

An even closer connection between scaling expressions and theory would be to express the global energy confinement time as a combination of confinement times representing the rore and the edge plasmas. In the core, for instance, there is some theoretical preconception and some supporting evidence that the confinement is controlled by two modes, the collisionless trapped electron mode and the ion temperature gradient, or $\eta_{i}$, mode for the electron and ion channels, respectively. (This is not meant as an endorsement of electrostatic processes for controlling transport; it is merely an example. Certainly in high- $B$ plasmas, which show similar confinement trends as low- $\beta$ plasmas, electromagnetic effects should be important. Largr-scale MHD processes also cannot be ignored.) The total confinenent in the core will be regulated by the faster of the above two parallel loss processes since the ions and electrons are coupled, so that

$$
\frac{1}{\tau_{E}^{c}}=\frac{1}{\tau_{E, e}^{c}}+\frac{1}{\tau_{E, z}^{c}}
$$

One might speculate that it is these core processes that cause the impfovement of confinement with ircreased density profile peakedness, as is seen in pellet and supershot 
discharges. The core confinement can be defined as

$$
\tau_{E}^{c}=\frac{\Delta W_{t h_{p \varepsilon}}}{\Delta P_{r c}}
$$

where $\Delta W_{t h_{r r}}$ is the excess energy stored inside $r_{\mathrm{r}}$ above some pedestal ("top of the pyramid" of stored energy), and $\Delta P_{r c}$ is the power inside $r_{c} . r_{c}$ is the radius separating the edge from the core; this radius is not yet clearly defined, but it may be, for instance, the $q=2$ surface.

The edge confinement time reflects the process controlling the effectiveness of the edge transport barrier, and may cause the $\mathrm{H}$-mode improvement and $J_{p}$ dependence. With the above prescription, the edge confinement time can be defined as

$$
\tau_{E}^{e}=\left(W-\Delta W_{t h e c}\right) / P_{\text {heat }}
$$

where $W$ is the total energy, $W-\Delta W_{\text {rc }}$ is the pedestal energy, and $P_{\text {heat }}$ is the total heating power. Since the edge and core processes are in series, the total confinement time is the sum of these two, and from the above definitions,

$$
\tau_{E}^{u_{h}}=W / P_{\text {heat }}=\tau_{E}^{e}+\frac{\Delta P_{r c}}{P_{\text {heat }}} \tau_{E}^{e},
$$

an expression which naturally leads to an offset linear form and which explicitly reflects heating deposition profile effects. It is possible that the small density dependence found in (3)-(4) and (10)-(12) may be due to variations in the heating deposition profile.

Plasma theory can also provide first principles gujdelines to help the experimentalist extract global scaling information from the data. ${ }^{48.49}$ The stored energy $\mathbb{I}^{*}=\int d^{3} \simeq \sum_{2}^{3} n_{j} T_{j}$ in an auxiliary heating experiment is additive, i.c.,

$$
W=W_{\text {oH }}+\int_{0}^{P_{\text {aux }}} \frac{d W}{d P_{\text {aux }}} d P_{\text {aux }}
$$

where

$$
\frac{d W}{d P_{\text {aux }}} \equiv \tau_{\text {inc }}
$$

is the incremental energy confinement time which may depend on $P_{\text {aur. }}$,

$$
\frac{W_{O H}}{P_{O H}(T)} \equiv \tau_{O H}
$$


is the ohmic confinement time to be evaluated at the presailing, not the initial, temperature. The global confin aent time $\tau_{E} \equiv \mathfrak{W} / P_{\text {loss }}$ becomes

$$
\tau_{E}=\frac{P_{O H} \tau_{O H}+\int_{0}^{P \ldots x} \tau_{\text {inc }} d P_{\text {aur }}}{P_{\text {aux }}+\left(P_{O H}+P_{\alpha}-P_{\text {rad }}\right)}
$$

where $P_{\text {lous }}$ denotes the transport losses which equal the total (net) input power

$$
P_{\text {co }}=P_{\text {aux }}+P_{\text {oH }}+P_{\alpha}-P_{\text {rad }}=P_{\text {lose }}
$$

Here, $P_{\alpha}$ would be the $\alpha$-particle heating power. Equation (17) reduces $\operatorname{tn} \tau_{E}=T_{O H}$ for $P_{a u x} / P_{O H} \rightarrow 0$, to "linear offset scaling" $\tau_{E}=\tau_{O H} \frac{P_{O H}}{P_{\text {Pur }}}-\tau_{\text {inc }}$ if $d \tau_{\text {irc }} / d P_{a u x}=0$ and $P_{\text {aux }} / P_{O H} \gg 1$ and to Goldston's formula if $\tau_{i n e} \propto P_{\text {aux }}^{-1 / 2}$ is used in (16) and $P_{a u x} / P_{O H} \gg 1$. Equation (17) contains the transition from ohmic to strongly heated scaling more naturally than Goldston's “inverse squares" data fit.

Thus, rather than trying to scale $\tau_{E}$, it may provide more insight to extract scaling information on the two more fundamental pieces $\tau_{O H}$ and $\tau_{\text {inc }}$ as a function of temperature $T$ and density $n$ or equivalently, as a function of $P_{a u x}$ and $n$. The function $T=T\left(P_{\text {aux }}\right)$ is known experimentally. (It is well known, e.g., that using $\langle n T\rangle / \tau_{E}=P_{\text {tot }}$ and Goldston's $\tau_{E} \sim P_{\text {tot }}^{-1 / 2}$ one finds $\tau_{E} \sim\langle n T\rangle^{-1}$.) Returning to Eq. (17), the various power terms in the denominator can be evaluated directly once the $T$ and $n$ profiles are known.

With the :sual assumption that the heat and particle fluxes consist of diffusive and convectjve pieces the local energy and particle balances for species $j$ are

$$
\begin{aligned}
-r \chi_{j} n_{j} T_{j}^{\prime}+r q_{c j} & =\int_{0}^{r} d r r S_{E_{j}} \\
-r D_{j} T_{j} n_{\jmath}^{\prime}+r \Gamma_{c j} T_{j} & =T \int_{0} d r r S_{p j} .
\end{aligned}
$$

Integrating by parts, the stored energy becomes

$$
\|=\sum_{j} W_{j}=\frac{3}{2} L \int_{0}^{a} d r r \sum_{j} n_{j} T_{j}=-\frac{3}{2} L \int_{0}^{a} d r \frac{r^{2}}{2} \sum_{j}\left(n_{j} T_{j}^{\prime}-n_{j}^{\prime} T_{j}\right)
$$

where $\mathrm{L}=4 \pi^{2} R$. Inserting $T_{3}^{\prime}, n_{3}^{\prime}$ from (19) into (20) produces three contributions to the stored energy" such that the global energy confineinent time becomes

$$
\tau_{E}=\frac{\sum W_{E j}+\sum W_{p j}-\sum W_{\mathrm{cj}}}{P_{\text {tot }}}
$$


where $P_{t o t}$ was defined in (18). $W_{E_{j}}$ depends on the transport quantities $\chi_{j}, S_{E_{j}}$ : $W_{p j}$ on $D_{j}, S_{p j}: W_{c j}$ on the convective $f u x$ pieces $q_{i j}, \Gamma_{c j}$. The source terms $S_{E j}$ correspond to those in Eq. (19). (The details of this derivation are contained in Appendix C of Sigmar and Hsu. ${ }^{49}$ ) Thus, the numerator in (21) depends on the local transport coefficients ${ }^{48}$ and comparing (21) with (17) reveals how $\tau_{O H}$ amd $\tau_{\text {anc }}$ are related to local transport. This formulation, then, produces a form for $\tau_{E}$ wh - ' vides a natural transition from $\tau_{O H}$ to $T_{\text {ineremental. The stored energy that goes into }}$ determining $\tau_{E}$ depends on four local quantities [see $\mathrm{Eq}$. (21)], indicating that energy and particle transport are intrinsically coupled in the plasma, and underscoring how misleading it can be to use simple estimates such as $\tau_{E} \simeq a^{2} / 4\left(\chi_{e}+\chi_{i}\right)$ without particle transport effects. Knowing the four transport quantities of $\mathrm{Eq} .(19)$ and temperature and density profiles allows $\tau_{E}$ to be uniquely determined from Eq. (20) and (21). For a given set of experimentally observed temperature and density profiles, this formalism is useful in quickly yielding the consequences for $\tau_{E}$ of a given theoretical model (i.e., for the transport quantities). Thus, comparison of the $\tau_{E}$ derived in this fashion to that determined from the experiment filters out the inappropriate lkat transport models.

An alternative approach to imposing theoretical constraints on GSEs is to test the scalings, or basic confinement trends, against the trends as derived from predictions of transport calculations. In this approach, a particular transport model is constructed from a combination of several theoretical transpu.rt coefficients; for example, large transport in the central sawtooth region combined with trapped electron and $\eta_{1}$ transport in the $1 \leq q \leq 2$ region and collisional transport outside $q-2$. Since a transport code is used, neoclassical transport rius the effects of neutrals (convective energy transport) and volume terms (radiation, charge exchange, heat depusition models, etc.) are included automatically. The code is run for all permutations of a range of machine parameters such as plasma current, density, heating power, and size. Each run of the code predicts plasma profiles and associated energy confinement time, and writes a record of these to a database. The predicted glubal scaling of $\tau_{E}$ based on these discharge variables may then be determined by doing a mul- 
tiple linear regression on the database of predicted $\tau_{E}$, exactly as is done with the experimental databases. The magnitude and scaling of $\tau_{E}$ derived from the model may then be compared with the experimental global scaling to decide whether the theoretical transport model thus constructed matches experiment. For this method to apply to models which depend on gradient scale lengths, more detailed comparison of predicted plasma profiles with experimental profile data would also be necessary.

\section{B. Relation to Particle Transport}

Particle confinement may be one of the least understood aspects of tokamak physics. As in the case of energy and momentum, particle transport is found to be highly anomalous with respect to neoclassical theory, at least in most plasma regimes. Particle and energy transport appear to be closely linked. This connection is most apparent when we consider the regimes of enhanced energy confinement. In all cases (H-Mode, pellet fueling, counter-injection, Z-mode, supershots, IOC) energy and particle confinement improve together. In the case of pellets, counter-injection, and IOC the improvement in particle confinement is accompanied by an improvement in ion energy confinement with fixed electron energy confinement. This observation, as well as the formalism in the previous section linking energy and particle confinement, puts clear constraints on the theories for anomalous transport. The transport of impurity particles follows the same trend and all the enhanced confinement regimes suffer from impurity build-up problems. It is also worth pointing out that at least in the ohmic regime, impurity confinement and electron energy confinem ${ }^{\circ}$ nt show markedly different scalings.

A crucial distinction must be made when we try to understand "global" scaling for particle transport. Unlike energy confinement which is dorinated by power sources located in the plasma core, global particle transport in most cases is dominated by particle sources localized in the plasma edge. The result is that global energy transport is a core quantity and global particle transport is an edge quantity. There is ample evidence that very different physics is dominating transport in the core and 
edge regions; therefore, we should not be comparing the two "global" quantities. With some care, particle transport coefficients can be obtained from "perturbation" experiments, such as oscillating gas puff or pellet injection. We need to compare these coefficients and their scalings to $\chi_{i}$ and $\chi_{e}$, which can also be measured similarly from localized transient responses. We also need to continue measurements of impurity transport in the plasma core and compare impurity transport and its scaling to transport of the background ions. Various impurity injection methods can be employed for this purpose. With this information in hand, it should be possible to develop a powerful "screen" for the transport theories. Theories which (after some adjustment of free parameters) can explain energy confinement must also explain particle and impurity transport. It should be pointed out that the transport coefficients derived from perturisation techniques are not necessarily jdentical to those in an unperturbed plasma. ${ }^{50}$ However, in most cases, the transport theories make unique predictions for particle and energy transport in the presence of these perturbations. Clearly, these are the comparisons to be made.

In order to improve our understanding of particle transport, we need to substantially broaden the data base for particle confinement. There are two approaches that can be taken. The first is to try to collect particle transport coefficients from the various machines as defined and derived by the local groups. A different approach would be to obtain data on the plasma profiles and sources and attempt to run identical analyses on all of the data. We should also be trying to define and suggest experiments that could be carried out on the existing and planned devices.

\section{Summary}

As $\tau_{E}$ is a fundamental, and easily determined, characteristic of tokamak discharges, it is necessary that we at least have an empirical understanding of the parametric dependence of this variable. The reasons for this necessity are 1) to aid in optimizing machine operation, and 2) to help guide theorist s and experimentalists in the important task of uncovering the physical processes that control transport and 
confinement.

In this report we have attempted to address several issues. foremost among them are the status and utility of presently existing L-mode scaling expressions, and how better and more appropriate descriptions can be developed. There are spveral djrections that future work on global scaling can take. The first, which is to fill the gaps in the present L-mode database, will require either additional experiments or refined analysis of existing information on plasma mass, elongation, and aspect ratio scalings. The second direction is a more sophisticated treatment of the data than has been done in the past, with particular emphasis on better estimates of the projection uncertainties for each scaling expression developed. Further work on scalings should also include studies of profile effects and an understanding of $\mathrm{H}$-mode and other enhanced confinement regime trends. A study of these regimes will require identification and quantification of a greater number of discharge characteristics than was necessary for L-modes.

Other approaches suggested in this report involved a re-direction of global scaling model assumptions, for they attempted to indicate ways in which scalings could be more intimately tied to theory. One of these is the use of appropriate dimensionless variables as scaling parameters. Another actually suggested dividing the plasma into edge and core regions, and developing confinement scaling descriptions for each regime separately. Finally, ways were suggested on how to input theoretical transport coefficients more directly into the scaling expressions themselves.

The new directions for global scaling studies will require a great dea] more work than that required for the relatively straight forward efforts in the past. The result of this new work, however, will be a more fundamental and accurate description of the parametric dependence of global confinement. 


\section{Acknowledgment}

A portion of this work was supported by the U. S. Dept. of Energy Contract No. DE-AC02-76-CHO-3073. 


\section{References}

'F. Perkins, in Heating in Toroidal Plasmas, volume 2, p. 977, Frascati, Italy, 1984, E.NEA, Proc. 4th Symp. Rome, 1984.

${ }^{2}$ W. Tang, C. Bishop, B. Coppi, et al., Microinstability based models for confinement properties and ignition criteria in tokamaks, in Plasma Physics and Controlled Fusion Research, volume 1, p. 337, Vienna, Austria, 1987, 1AEA, Proc. 11th Int. Conf. Kyoto, 1986.

${ }^{3}$ R. Dominguez and R. Waltz, Nucl. Fusion 27, 65 (1987).

4J. Kesner, Nucl. Fusion 29 (1988).

${ }^{5}$ D. Jassby, D. Cohn, and R. Parker, Nucl. Fusion 16, 1045 (1976).

${ }^{6} \mathrm{~J}$. Hugill and J. Sheffield, Nucl. Fusion 18, 15 (1978).

'W. Pfeiffer and R. Waltz, Nucl. Fusion 19, 51 (1979).

${ }^{8}$ R. Parker, M. Greenwald, S. Luckhardt, E. Marmar, M. Porkolab, and S. Wolfe, Nucl. Fusion 25, 1127 (1985).

${ }^{9}$ R. Goldston, Plasma Phys. Controlled Fusion 26, 87 (1984).

${ }^{10}$ S. Kaye, Phys. Fluids 28, 2327 (1985).

${ }^{13}$ S. Kaye and R. Grsldston, Nucl. Fusion 25, 65 (1985).

${ }^{12} V$. Merezhkin and V. Mukhovatov, Sov. JETP Lett. 33, 446 (1981).

${ }^{13} \mathrm{Y}$. Esspchuk and the T-10 Group, Investigation of energy confinement in ECRH experiments on T-10, 1988.

${ }^{14}$ P. Rebut, P. Lallia, and M. Watkins. The critical temperature gradient model of plasma transport, Proc. 12th Int. Conf. Nice, 1988. IAEA-CH-50/D-4-I, 1988.

${ }^{15}$ II. Watkins, private communication, 1988. 
${ }^{16} Y$. Shimomura and K. Odajima, Energy confinement scaling based on offset linear characteristic, private communication, 1988.

${ }^{17}$ H. Murmann, F. Wagner, and the ASDEX-, NI-, ICRH-teams, The isotope dependence of global confinement in ohmically and auxiliary heated ASDEX plasmas, in Controlled Fusion and Plasma Heating, volume 1, p. 3, EPS, Proc. 15th Eur. Conf. Dubrovnik, 1988, 1988.

${ }^{18}$ J. DeBoo, K. Burrell, S. Ejima, el al., Nucl. Fusion 26, 211 (1986).

${ }^{29}$ N. Suzuki, Y. Miura, M. Hasegawa, et al., in Controlled Fusion and Plasma Physzcs, volume 2, p. 217, EPS, Proc. 14th Eur. Conf. Madrid, 1987, 1987.

${ }^{20} \mathrm{~J}$. Cordey, D. Bartlett, V. Bhatnager, et al., in Plasma Physics and Controlled Fusion Research, volume 1, p. 99, Vienna, Austria, 1987, IAEA, Proc. 11th lnt. Conf. Kyoto, 1986.

${ }^{21}$ D. Schissel, K. Burrell, J. DeBoo, et al., Nucl. Fusion 29, 185 (1989).

${ }^{22} \mathrm{O}$. Kardaun, private cornmunication, 1988.

${ }^{23}$ M. Nagami, M. Kasai, A. Kitsunesaki, et al., Nucl. Fusion 24, 183 (1984).

${ }^{24}$ F. Wagner, G. Becker, K. Behringer, et al., Phys. Rev. Lett. 49, 1408 (1982).

${ }^{25}$ S. Kaye, M. Bell, K. Bol, et al., J. Nucl. Mater. 121, 115 (1984).

${ }^{26} \mathrm{~K}$. Burrell and the Doublet-J] Group, in Controlled Fusion and Plasma Physzes, volume 1, p. 11, Linnich. 1983, EPS, Proc. 11th Eur. Conf. Aachen, 1983.

${ }^{27}$ K. Burrell, S. Ejima, D. Schissel, et al.. Phỵs. Rev. Lett. 59, 1 I5 (1987).

${ }^{28}$ A. Tanga, D. Bartlett, K. Behringer, et al., in Plasma Physıcs and Controlled Fuston Restarch. volume 1, p. 65, Vienna, Austria, 1987, IAEA. Proc. 11th Int. Conf. Kyoto, 1986. 
${ }^{29}$ K. Odajima, A. Funahashi, K. Hoshino, et al.. in Plasma Physics and Controlled Fusion Research, volume 1, p. 151, Vienna. Austria, 1987, IAEA, Proc. lith Int. Conf. Kyoto, 1986.

${ }^{30}$ E. Lazarus, J. Bell, C.. Bush, et al., J. Nucl. Mater. 121, 6] (1984).

${ }^{31}$ S. Milora, C. Foster, C. Thomas, et al., Nucl. Fusion 20, 1491 (1980).

${ }^{32}$ M. Greenwald, D. Gwinn, S. Milora, J. Parker, R. Parker, et al., Phys. Rev. Lett. 53, 352 (1984).

${ }^{33}$ S. Wolfe, M. Greenwald, R. Gandy, R. Granetz, C. Gomez, et al., Nucl. Fusion 26, 329 (1986).

${ }^{34}$ G. Schmidt, S. Milora, V. Arunasalam, et al., in Plasma Physics and Controlled Fusion Research, volume 1, p. 171, Vienna, Austria, 1987, IAEA, Proc. 11th Int. Conf. Kyoto, 1986.

${ }^{35}$ M. Kaufmann, K. Buchl, G. Fussmann, et al., Nucl. Fusion 28, 827 (1988).

${ }^{36} \mathrm{G}$. Schmidt and the JET Team, Heating of peaked density profiles produced by pellet injection in JET, Proc. 12th Int. Conf. Nice, 1988, IAEA-CH-50/A-IV-I, 1988.

${ }^{37}$ J. Strachan, M. Bitter, A. Ramsey, et al., Phys. Rev. Lett. 58, 1004 ( 1987 ).

${ }^{38}$ E. Muller. F. Soldner, G. Janeschitz, et a]., Improved confinement at high densities in ohmically heated and gas refuelled divertor discharges in ASDEX. in Controlled Fusion and Plasma Heating, volume 1, p. 19, EPS, Proc. 15th Fur. Conf. Dubrovinik, 1988, 1988.

${ }^{39} \mathrm{~S}$. Tsuji, M. Akiba, T. Ando, et al. Energy confinement with auxiliary heating in JT-60, Proc. 12th Int. Conf. Nice, I988, IAEA-CH-50/A-V-1, 1988.

40J. Manickam. V. Arunasalam, M. Bel], et al., Proc. 12tl Int. Conf. Nice. 1988, IAEA-C'H-50/A-VII. \&, 1988. 
"'D. Schissel. X. Bronks. K. Burrell, et al.. Confinement scaling studies in DIII-D, in Controlled Fuszon and Plasma Phystcs, volume 1, p. 115. EPS. Proc. 16th Eur. Conf. Venice. 1989. 1989.

${ }^{42} \mathrm{~K}$. Fiedel. Advanced statistics for tokamak transport colinearity and tokamak to tokamak variation. Xuclear Fusion (to be published).

${ }^{43}$ B. Tichelaar and L. Huff, EOS 70, 593 (1989).

${ }^{44}$.M. Sugihara and C. Singer, Nucl. Fusion 26, 1547 (1986).

${ }^{45}$ H. Park. Phys. Rev. Lett. (to be published).

${ }^{46} \mathrm{~J}$. Connor, Invariance princjples and plasma confinement, private communication, 1987.

${ }^{47}$ T. Stix, Plasma Phys. 14, 367 (1972).

${ }^{48}$ J. C'allen. J. Christiansen, J. Cordey. P. Thomas, and K. Thomsen. Nucl. Fusion 27. 1857 (1987).

${ }^{49}$ D. Sigmar and C. Hsu. Comments Plasma Phys. Controlled Fusion 12. 15 (1988).

${ }^{50}$ E. Fredrickson. .J. C'allen. K. McGuire, et al.. Nucl. Fusion 26. 849 (1986). 


$\begin{array}{lrl}\text { MACHINE } & \text { DISCHARGES } & \text { ADDITIONAL CONSTHAINTS } \\ \text { ASDEX } & 32 & \text { L-mode only } \\ \text { DITE } & 205 & \\ \text { DIII } & 212 & \text { Limiter only } \\ \text { ISX-B } & 108 & \\ \text { PDX } & 46 & \text { Limiter only } \\ \text { PLT } & 70 & \\ \text { TFR } & 4 & \\ \text { JFT-2M } & 112 & \text { Limiter only } \\ \text { JET } & 593 & \text { Limiter, inner wall only } \\ \text { TFTR } & 189 & \\ \text { JT-60 } & 212 & \text { Limiter only for } 2.5-2.8 \text { MA } \\ \text { DHII-D } & 16 & \text { L-mode only }\end{array}$

Table I: Global L-mude Database 


\begin{tabular}{|c|c|c|c|c|c|c|c|c|c|}
\hline MACIINE & $a(m)$ & $R(m)$ & 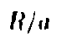 & $r$ & $B_{1}(T)$ & $I_{1}(M / 1)$ & $\dot{n}_{e}\left(10^{19} m^{3}\right)$ & $\left.\operatorname{limas}_{111,}(.1 / 1)^{2}\right)$ & Comuments \\
\hline THIR & $0.17-0.19$ & $0.96 ;-11.97$ & 5.4 & 1.0 & 3.7 .1 .1 & $0.23-10.30$ & $8.7 \cdot 12.9$ & 1.1 & N|3| \\
\hline ISX.13 & $0.25-0.27$ & $0.92 \cdot 0.95$ & 3.6 & $1.0 .5-1.5 .4$ & $0.7-1.5$ & $0 .(06-1) .21$ & $1.5 \cdot 1.3 .2$ & 2.1 & NBI \\
\hline 1)TH: & 0.15 .0 .25 & $1.000-1.29$ & 5.7 & 1.00 & $0.9-0.2 T$ & $0.107-0.26$ & 1.0 .9 .9 & 2.0 & N[3] \\
\hline.$[M] \cdot 2 N$ & $0.32-0.34$ & $1.28-1.30$ & 3.9 & $1.3 \cdot 1 \cdot 1.52$ & $1.0-1.3$ & $0.16-0.35$ & 1.6-4. 7 & 2.7 & NBI, IC'KF \\
\hline Pין & 0.111 & 1.31 & 3.4 & 1.0 & $2.0-3.3$ & $0.26-0.58$ & 1.26 .3 & 3.1 & NB3I \\
\hline 1111 & $0.311-0.41$ & $1.12-1.13$ & 3.7 & $0.95-1.79$ & $0.6-2.1$ & $0.23-0.81$ & $2.0-9.9$ & 4.9 & $\mathrm{NHI}$ \\
\hline איוי & $0.12 \cdot 0.1 .1$ & 1.43 & 3.3 & 1.0010 & $0.7-2.2$ & $0.23-0.48$ & $2.1-7.0$ & 5.5 & $\mathrm{NHI}$ \\
\hline ASIIE:X & $0.11\}$ & 1.65 & 1.1 & 1.010 & 2.2 & $0.20-0.38$ & $2.7-5.9$ & 2.3 & NI3I \\
\hline |1III. [1 & 0.60 .0 .64 & 1.70 & 2.7 & $1.70 \cdot 1.80$ & $1.8-2.1$ & 1.00 & $1.5-3.3$ & 6.1 & $N|3|$ \\
\hline TFlK & $0.79 \cdot 0.8 .1$ & $2.45 \cdot 2.57$ & 3.0 & 1.00 & $1.0-5.2$ & $0.9(1)-2.51$ & $2.5 \cdot 7.4$ & 20.1 & NI3I \\
\hline JT 60 & $\left.0.6 .1-0.0^{\prime}\right]$ & $2.96 \cdot 3.11$ & 3.5 & $0.84 \cdot 1.01$ & $.01 .-1.8$ & 1.000 .3 .18 & 1.0 .11 .9 & 23.1 & NHI, ICKK. III \\
\hline IFH & $1.12 \cdot 1.212$ & $2.8 .3 \cdot 2.96$ & 2.5 & $1.333-1.66$ & 1.7 .3 .5 & $0.00-5.0 .1$ & $0.8-4.8$ & 15.1 & N|3], IC'BF' \\
\hline
\end{tabular}

Table Il: Machins (Dperating Parameters 


$\begin{array}{lccccc}\text { MACHINE } & \underline{\kappa} & \underline{I_{p}} & \underline{\bar{n}_{e}} & \underline{B_{t}} & \underline{\underline{P_{\text {heat }}}} \\ \text { ISX-B } & 0.00 & 1.51 & 0.09 & -0.11 & -0.67 \\ \text { DIII } & 0.46 & 0.86 & 0.08 & 0.32 & -0.66 \\ \text { PDX } & & 1.07 & 0.27 & -0.08 & -0.30 \\ \text { ASDEX } & & 1.11 & -0.27 & & -0.27 \\ \text { DIII-D } & & & & & -0.67 \\ \text { JFT-2M } & & 0.70 & 0.38 & & -0.59 \\ \text { TFTR } & & 0.81 & & & -0.47 \\ \text { JT-60 } & & 0.69 & 0.13 & -0.46 & -0.47 \\ \text { JET } & & 0.87 & 0.08 & 0.33 & -0.56\end{array}$

Table 11l: Regression Coefficients 


\section{Figures}

FIG. 3. Range of plasma current as a function of aspect ratio for standard operating regimes of previous and present on-line tokamak experiments.

FIC. 2. Experimental is. fit $\tau_{E}$ values for the three recent scalings described in the text, all-simple (a), all-complex (b), and big (c).

FIG. 3. Comparison of experimental confinement times and those predicted by various scaling expressions for JET power scans at 1, 3, and 5 MA. Further operational details of these scans are given in the text. The scaling expressions used in these comparisons are Kaye-Goldston (a), modified Kaye-Goldston (b), GoldstonAachen (c). Rebut-Lallia (d), Shimomura-Odajima (e), Merezhkin-Mukhovatov (f), T-10 (g), all-simple (h), all-complex (i). and big (j).

FIC. 4. Root mean square prediction error of the ten global scaling expressions for all data in the L-mode database (with the data constrained to low density as prescribed in the text for Merezhkin-llukhovatov).

FIG. 5. Incrementa] confinement time as a function of plasma current for five tokamak experiments. The incremental confinement time was determined from the slupe of stored energy vs. heating power at each current for each machine listed. Nite that values of the incremental confinement time and plasma current are multiplied by a factor of ten for PDX and DIIl for graphical purposes. 


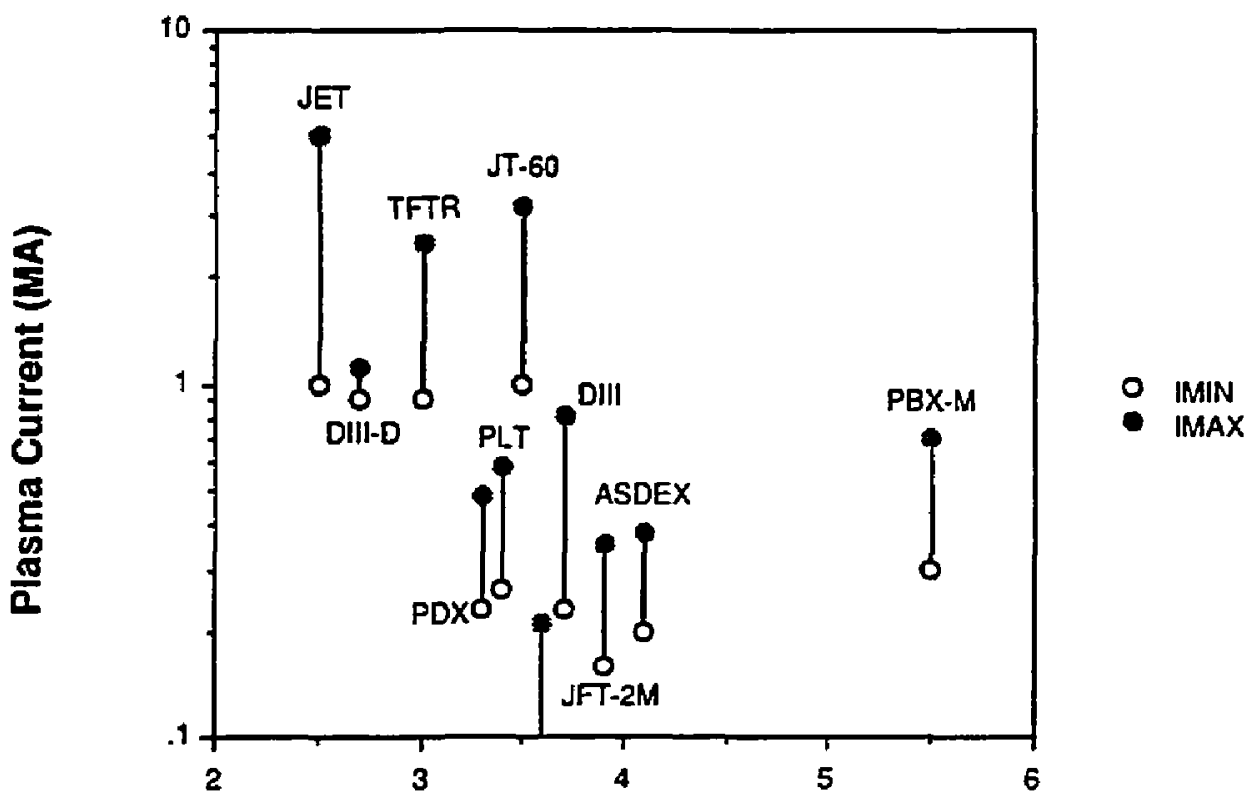

R/a

Figure 1 


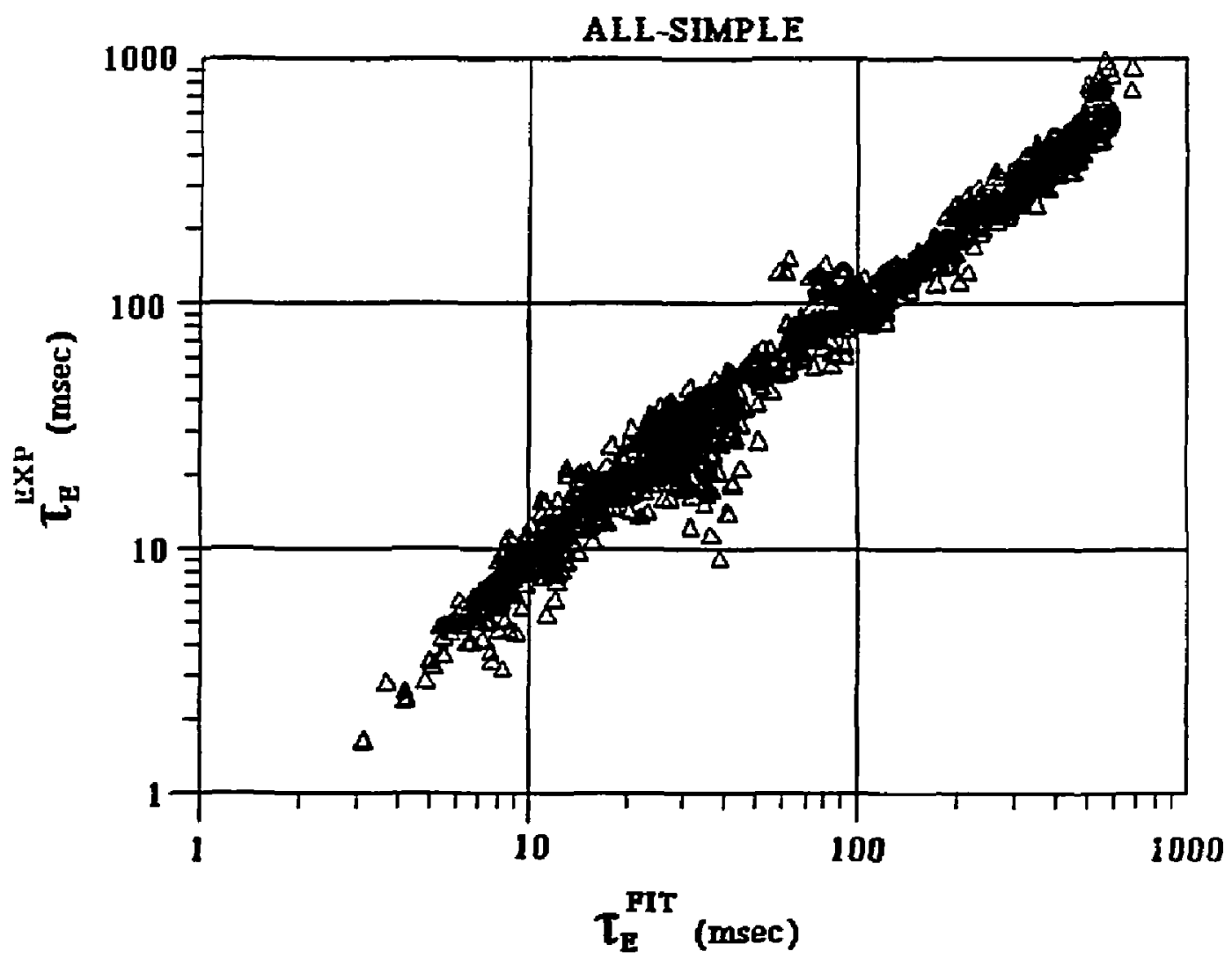

Figure 2a 


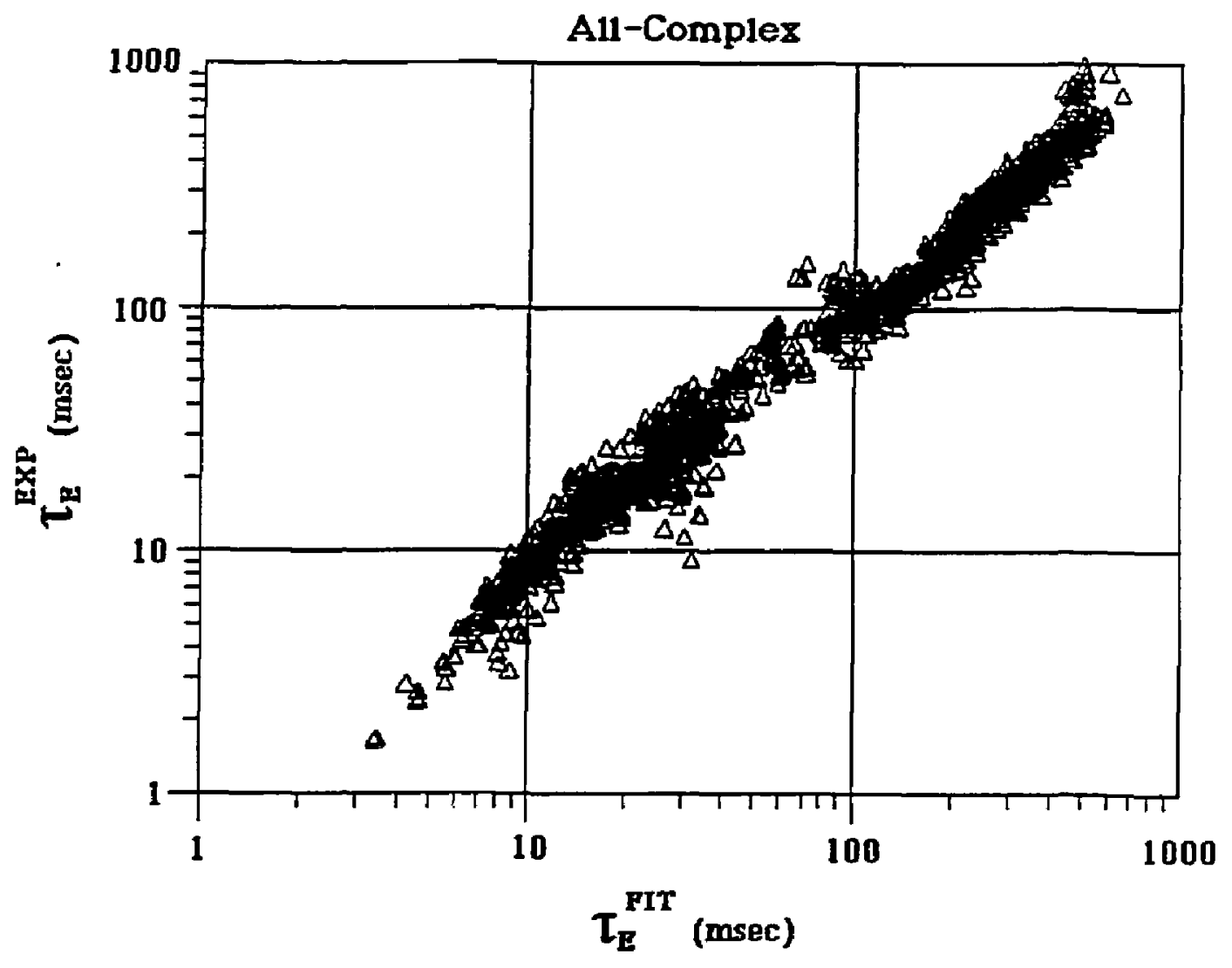

Figure 2b 


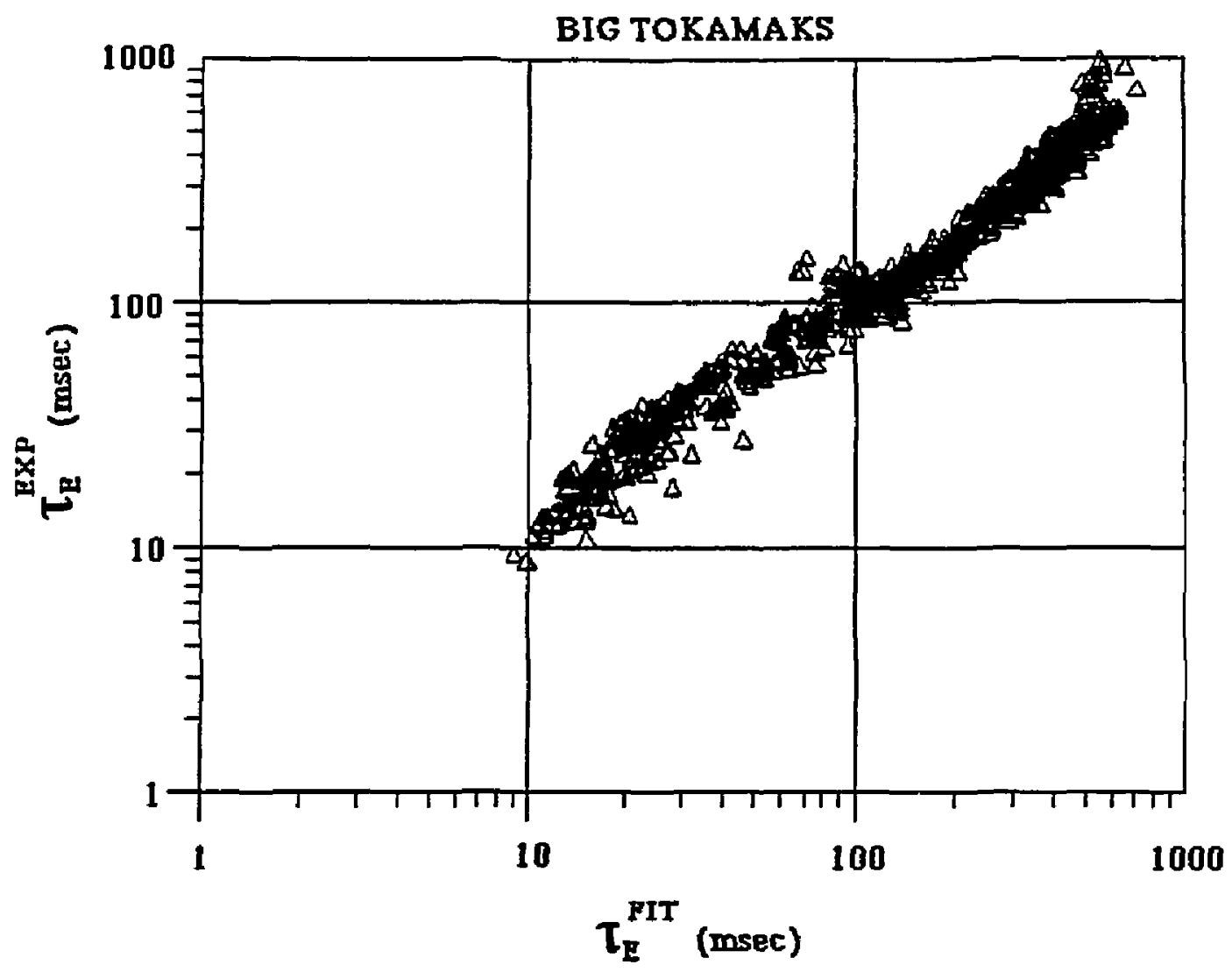

Figure 2c 


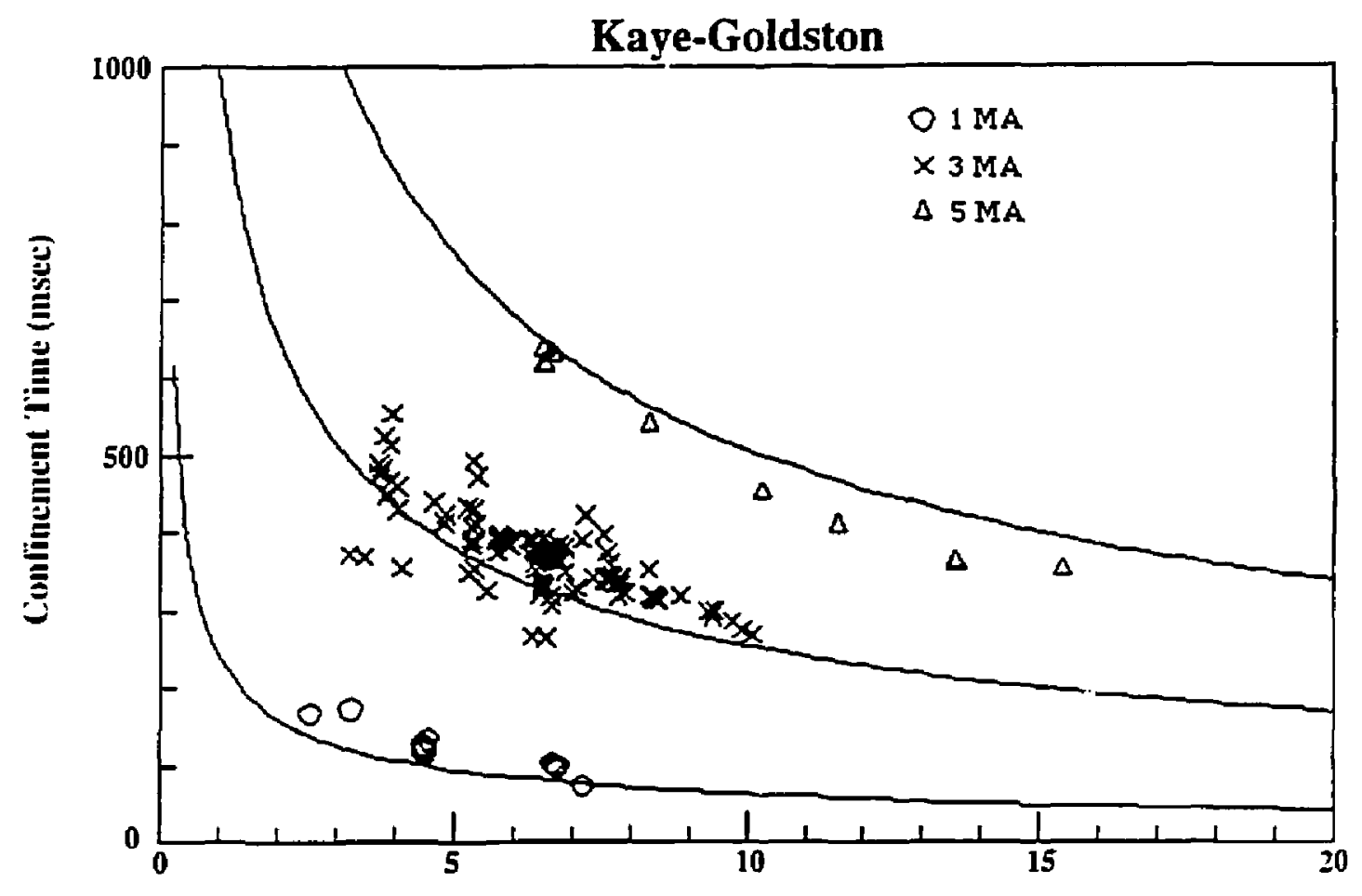

Heating Power (MW)

Figure $3 a$ 


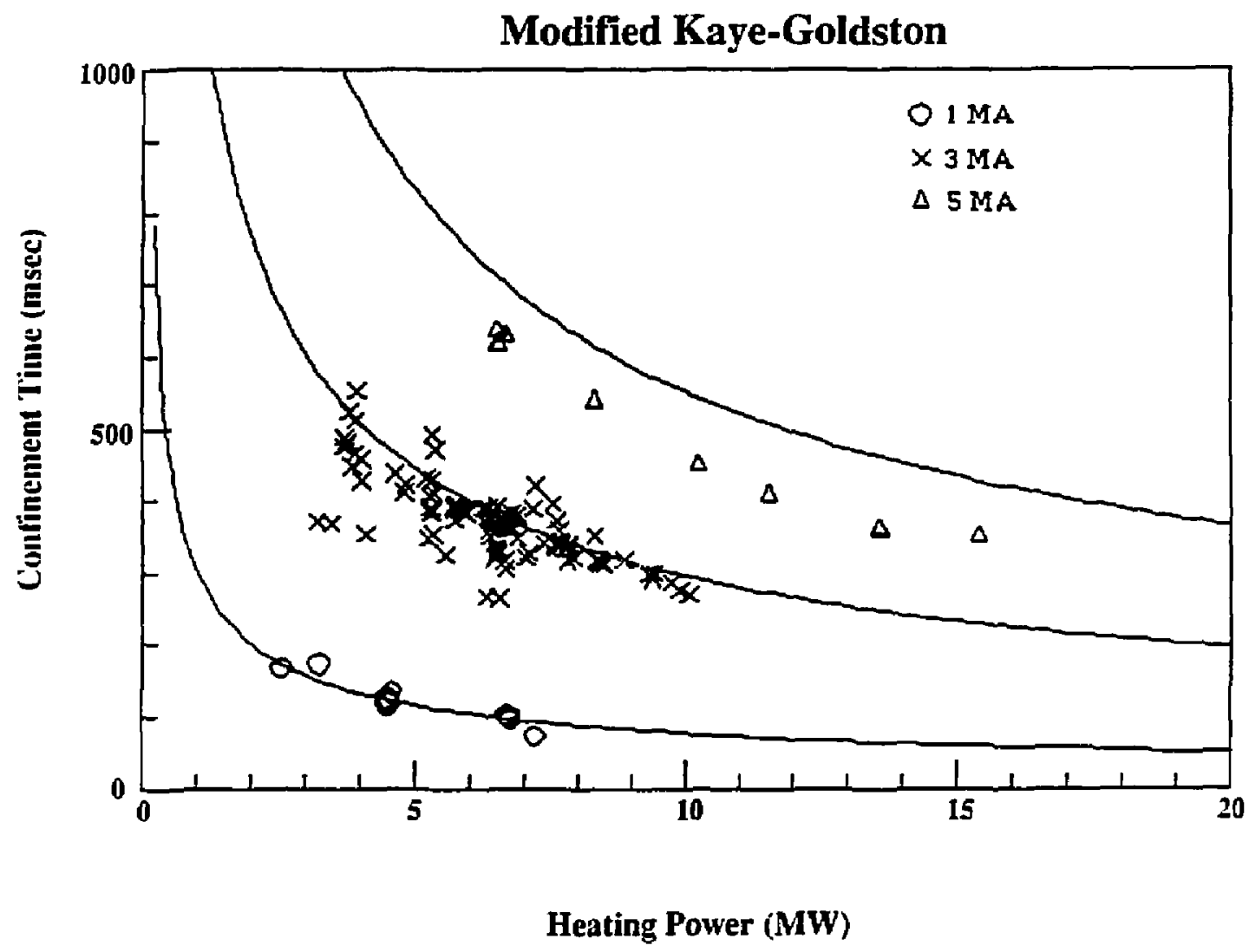

Figure 3b 


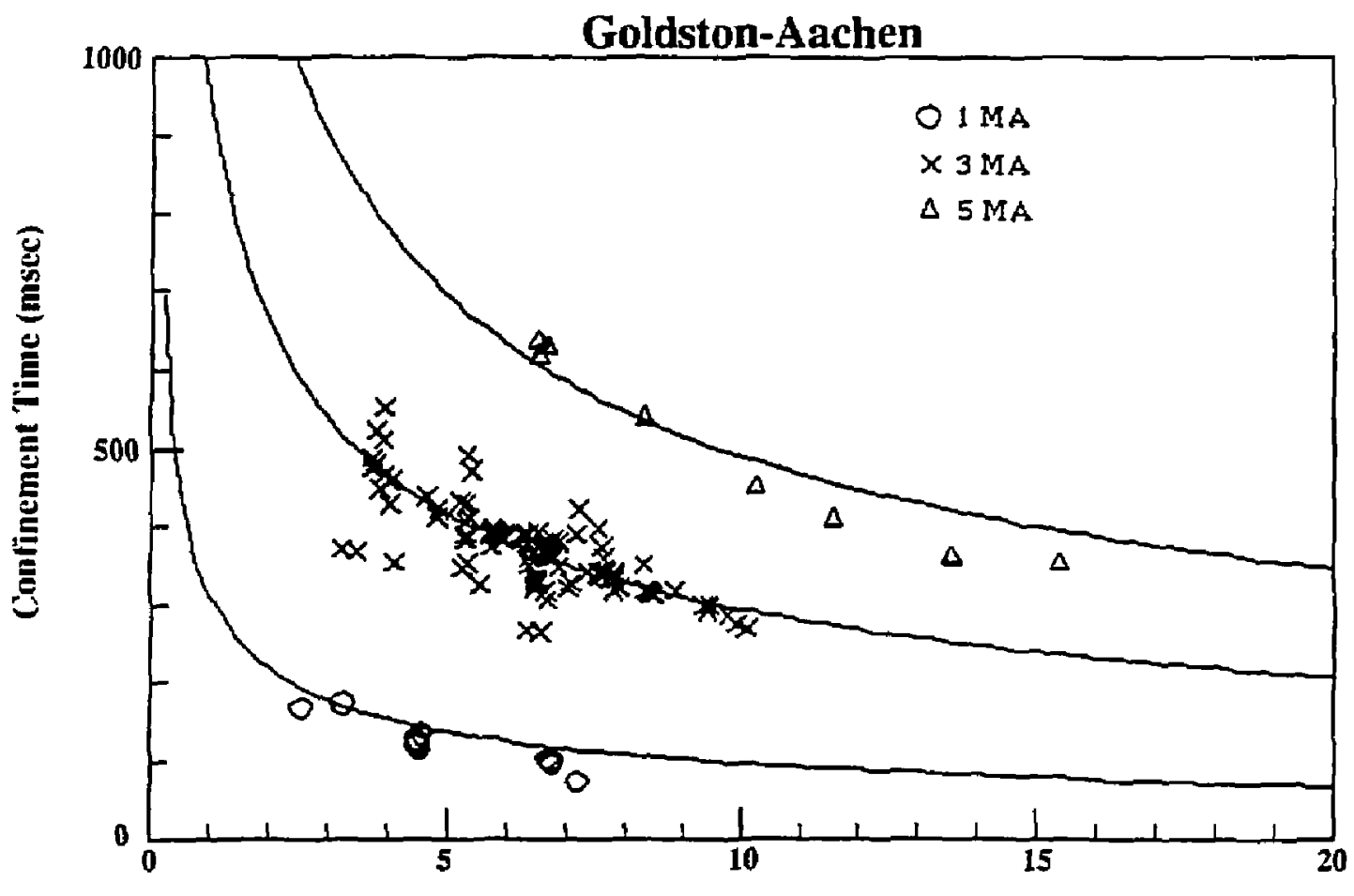

Heating Power (MW)

Figure 3c 


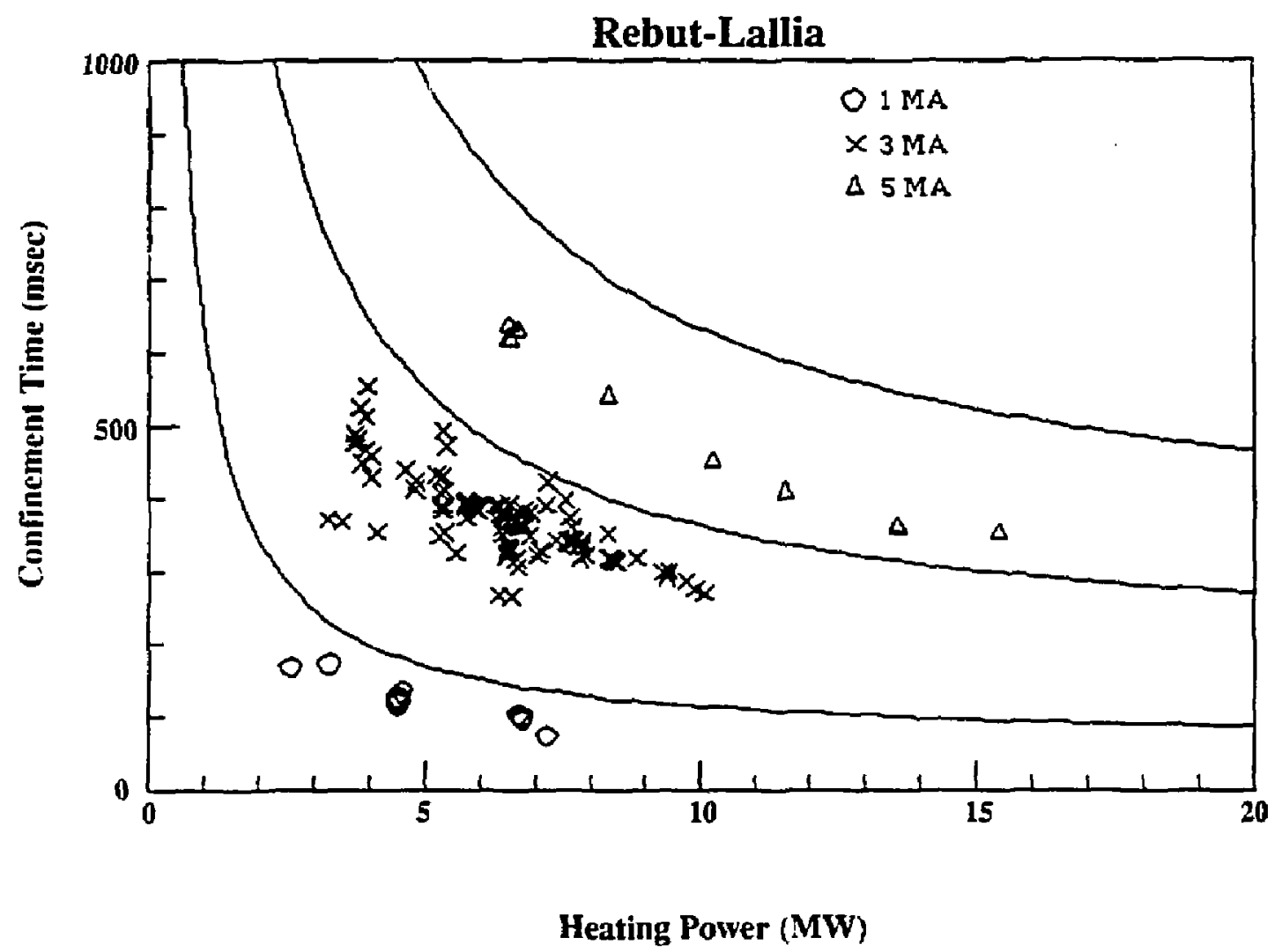

Figure $3 d$ 


\section{Shimomura-Odajima}

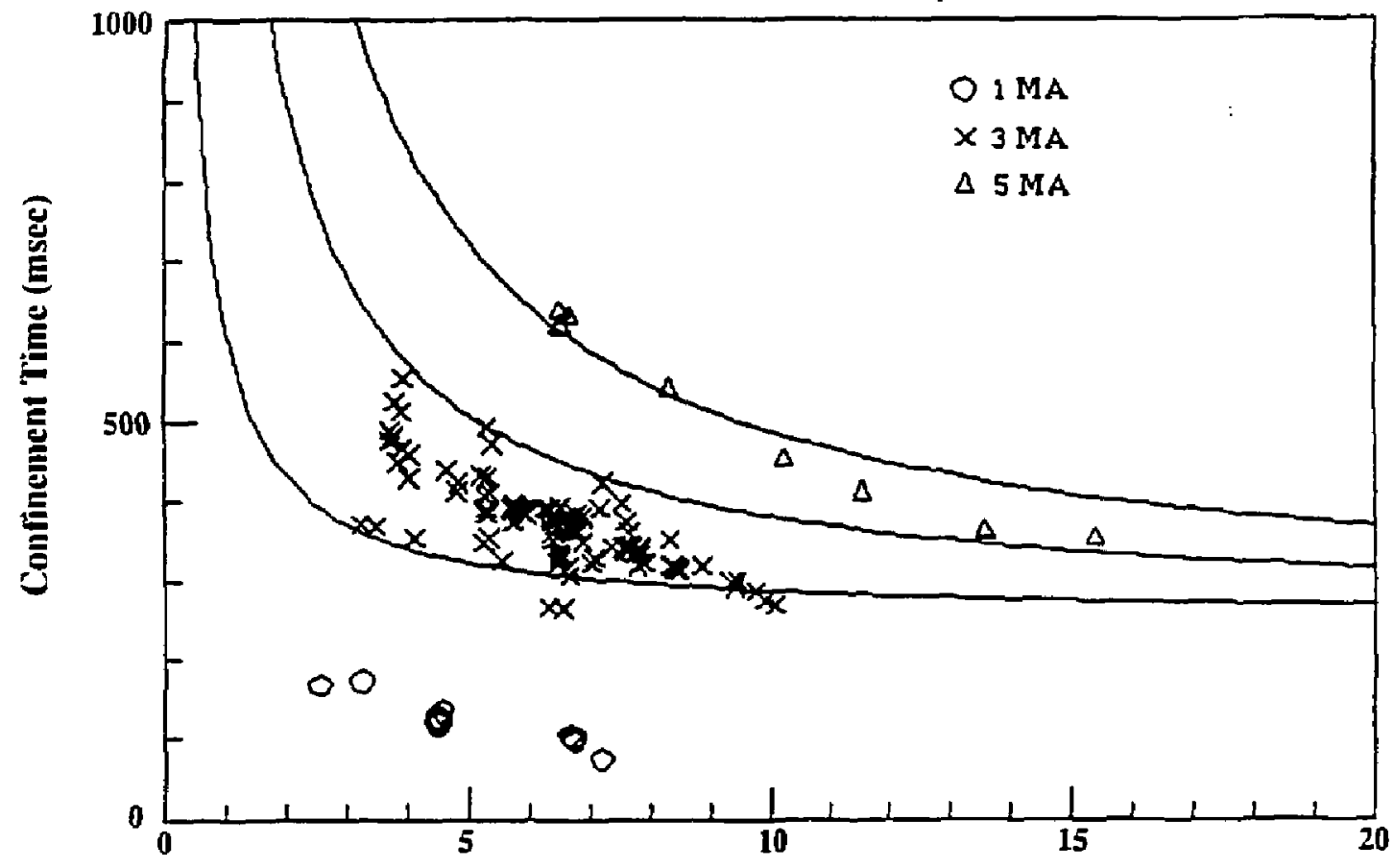

Heating Power (MW)

Figure $3 e$ 


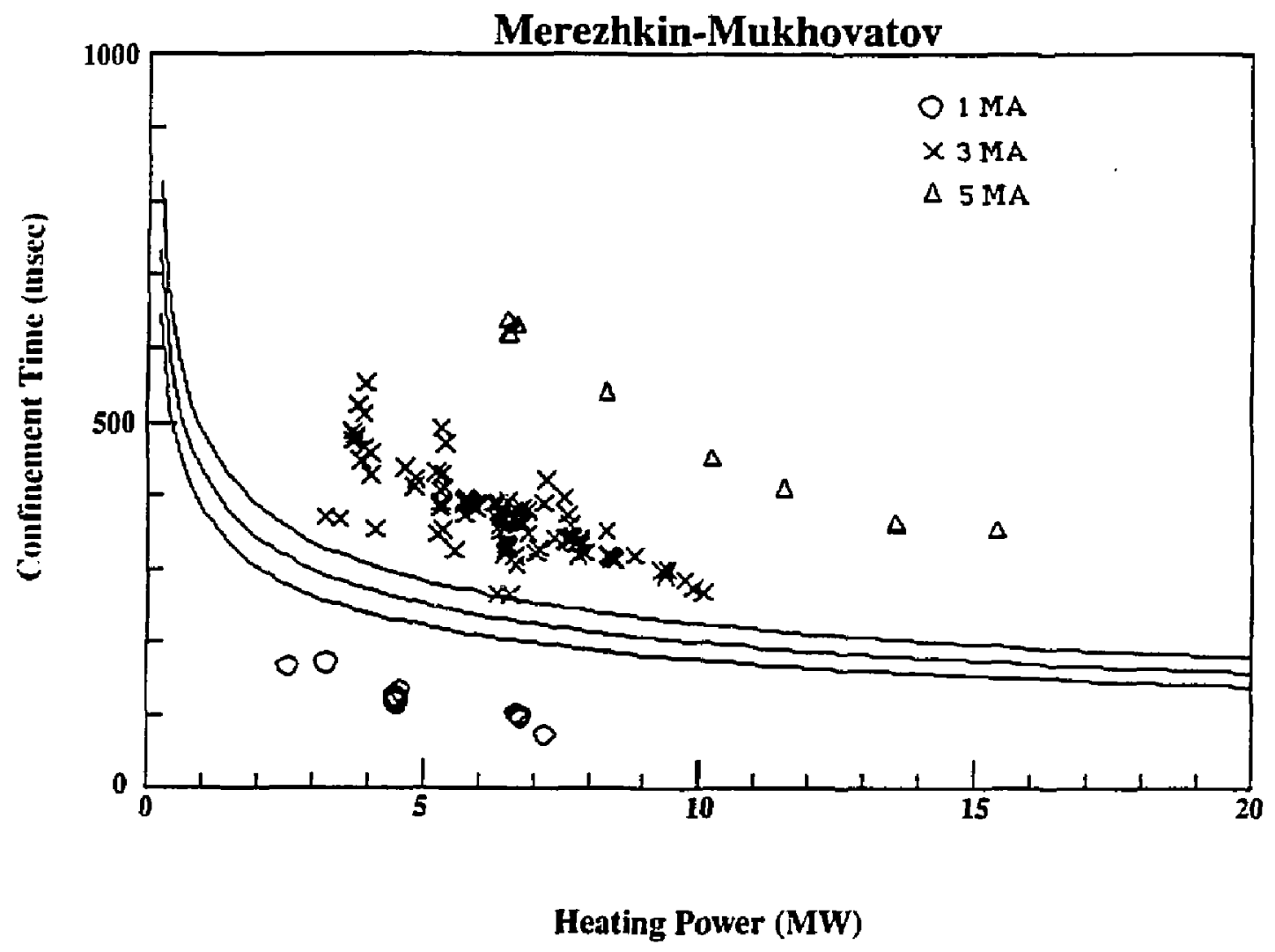

Figure $3 f$ 


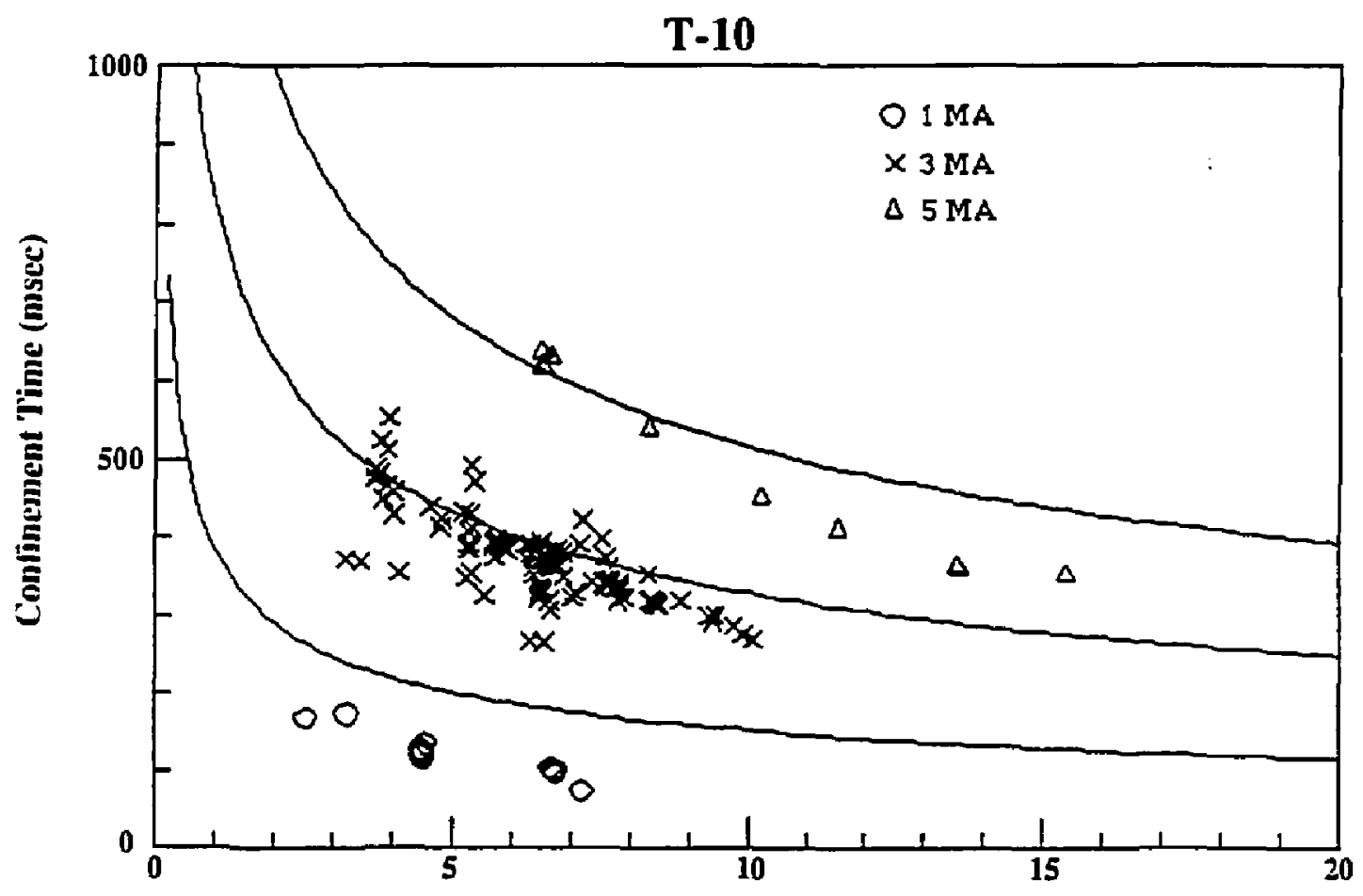

Heating Power (MW)

Figure $3 g$ 


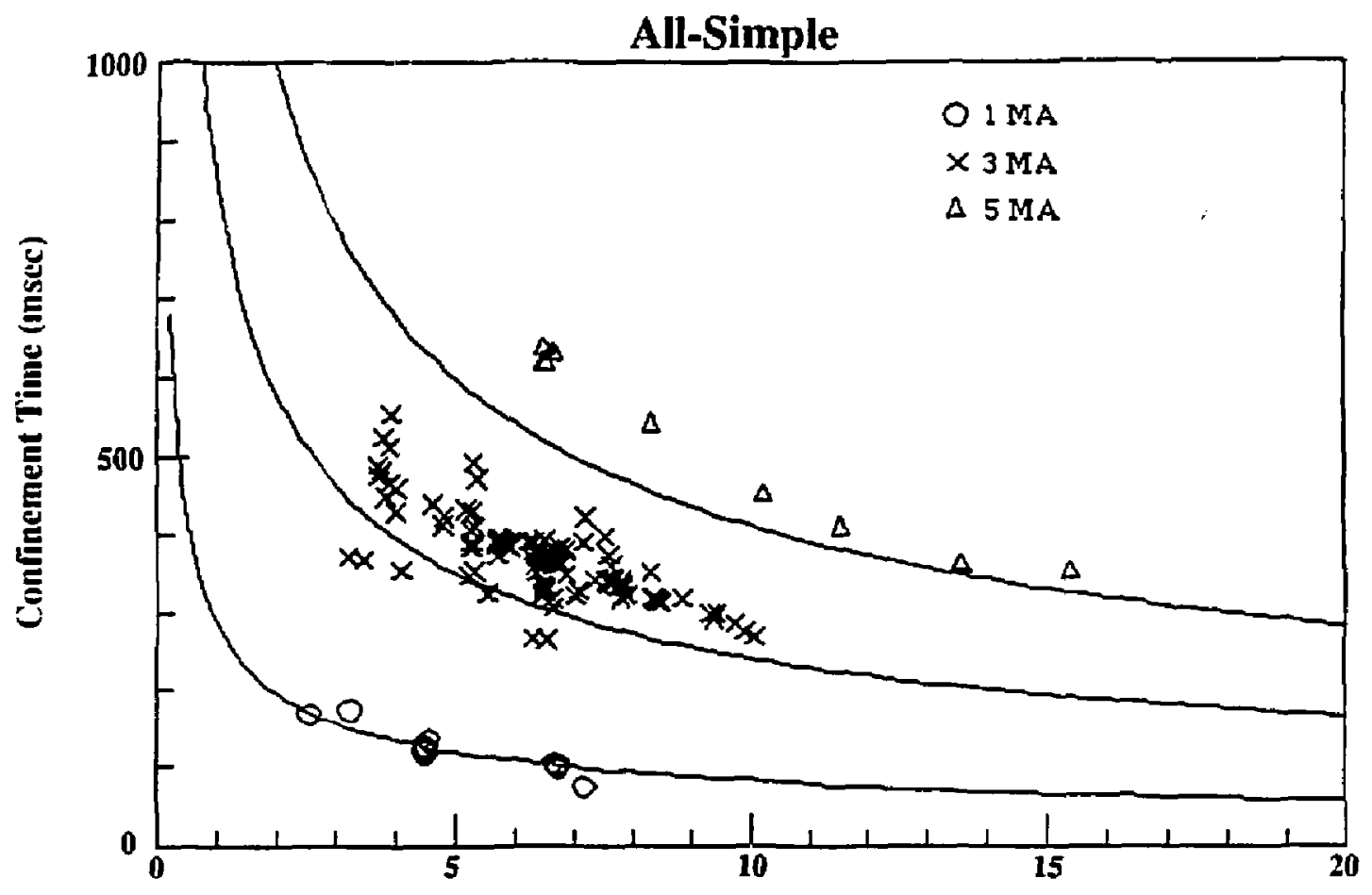

Heating Power (MW)

Figure 3h 


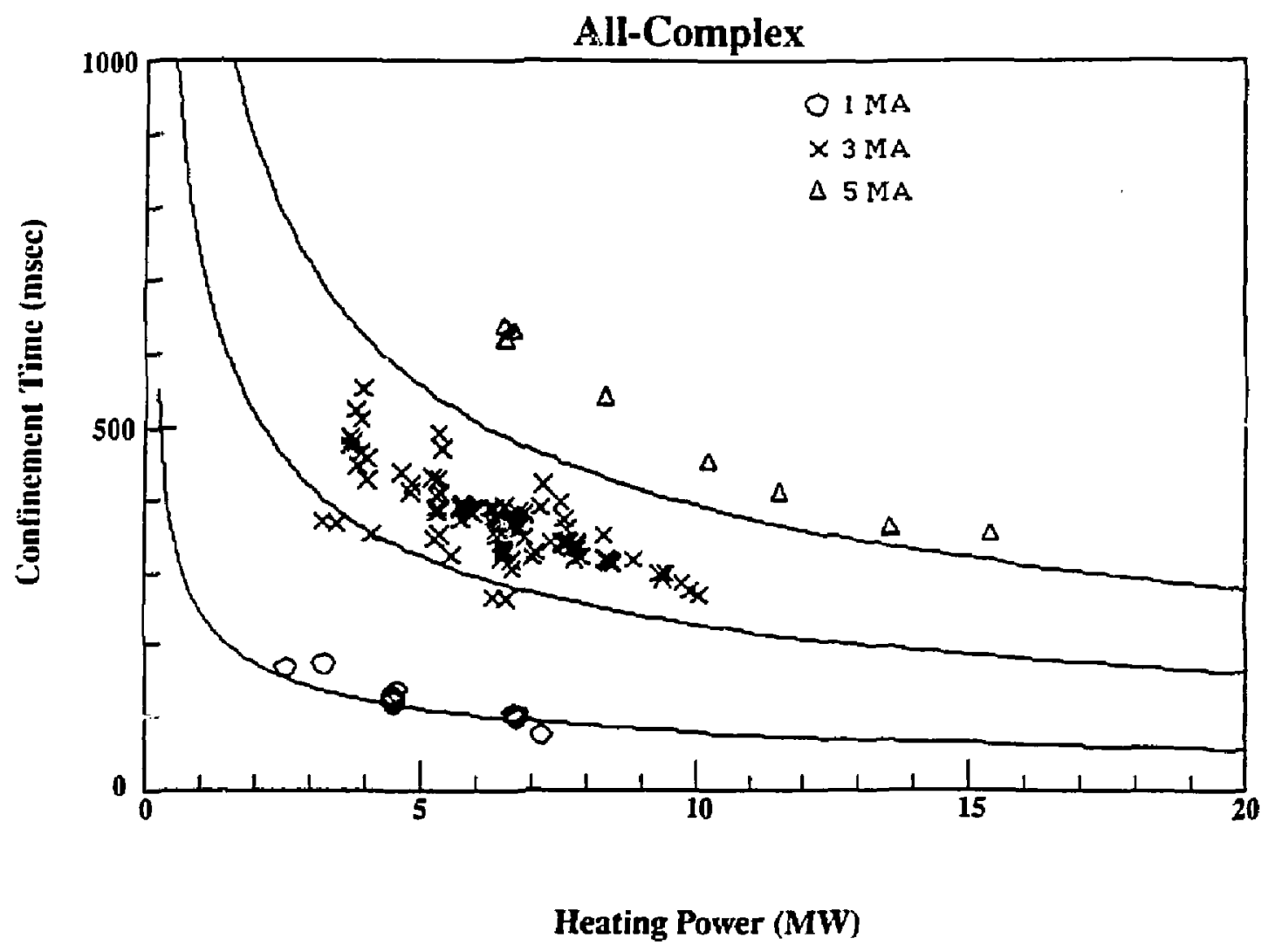

Figure 3i 


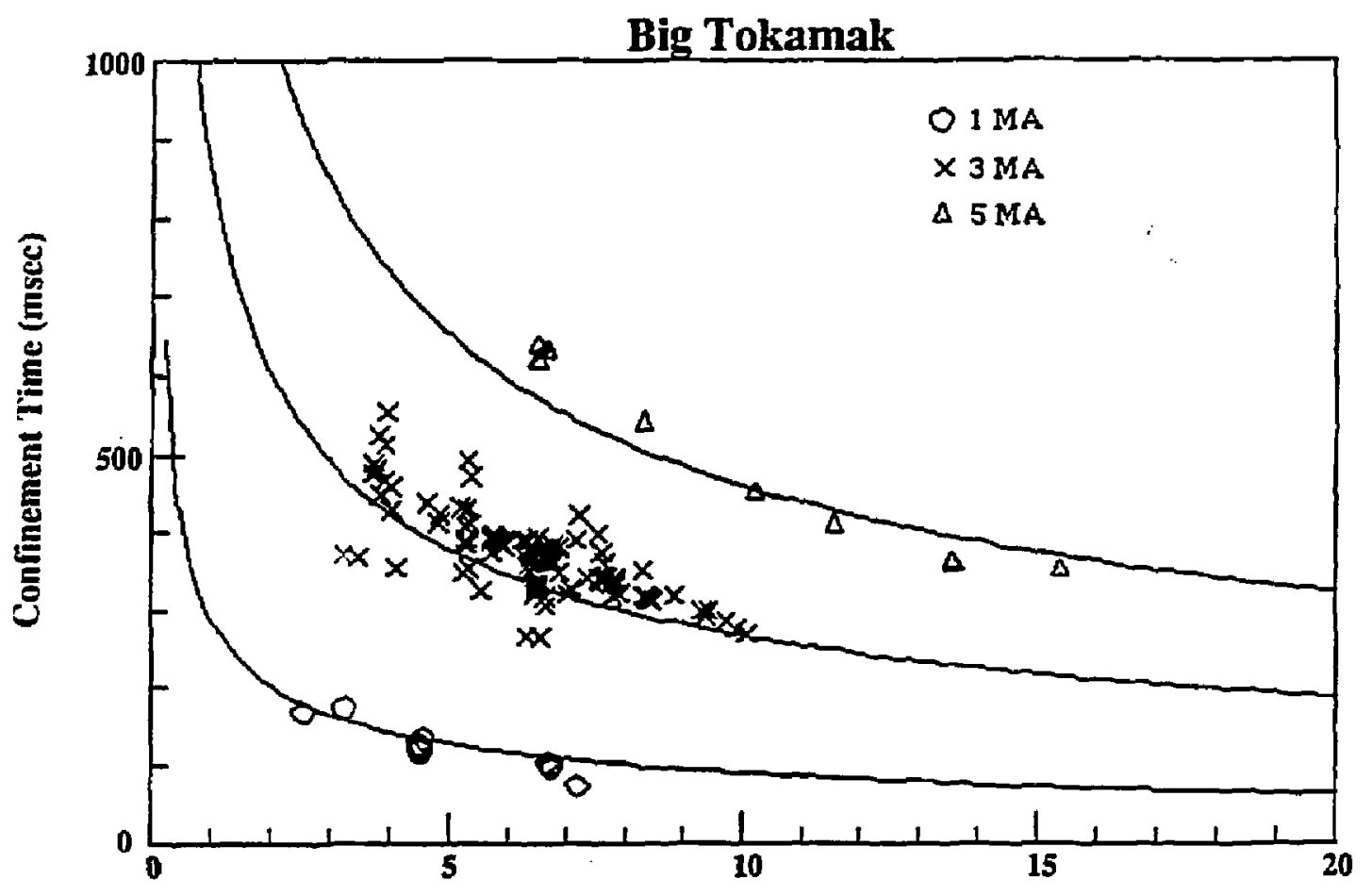

Heating Power (MW)

Figure 3j 
All Machines

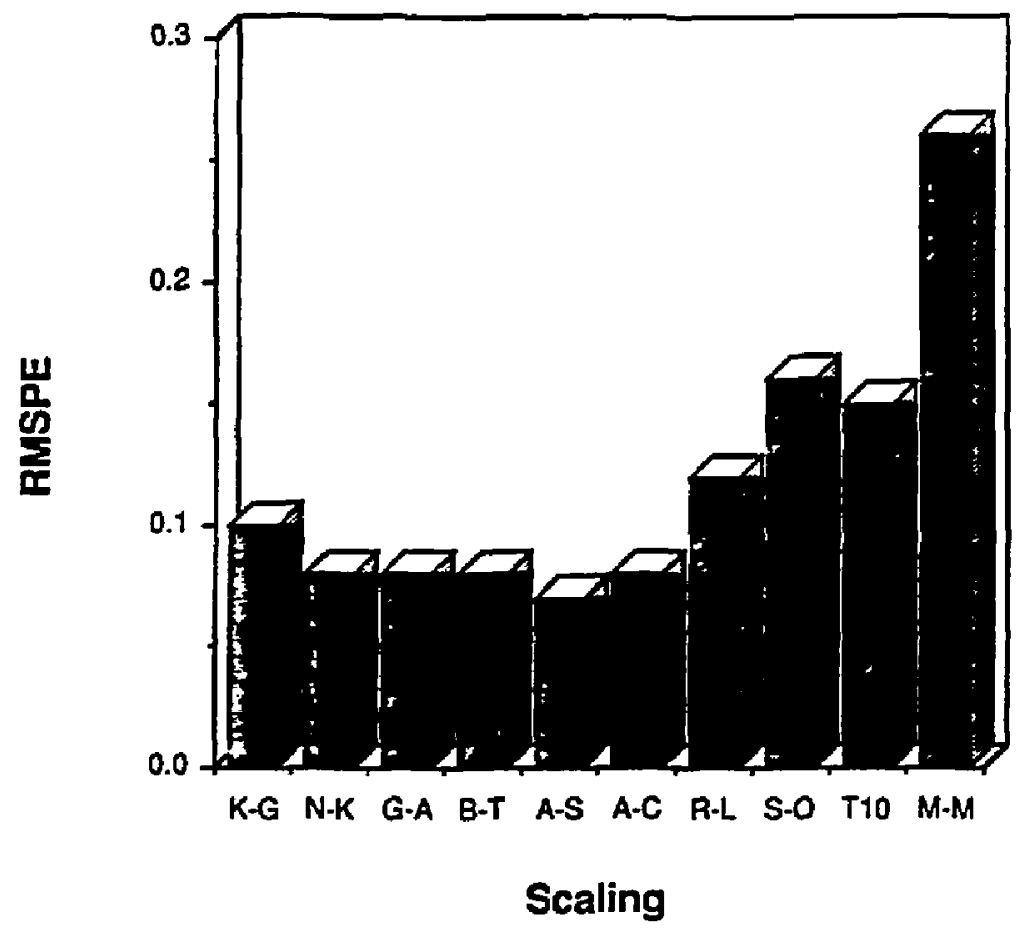

Figure 4 


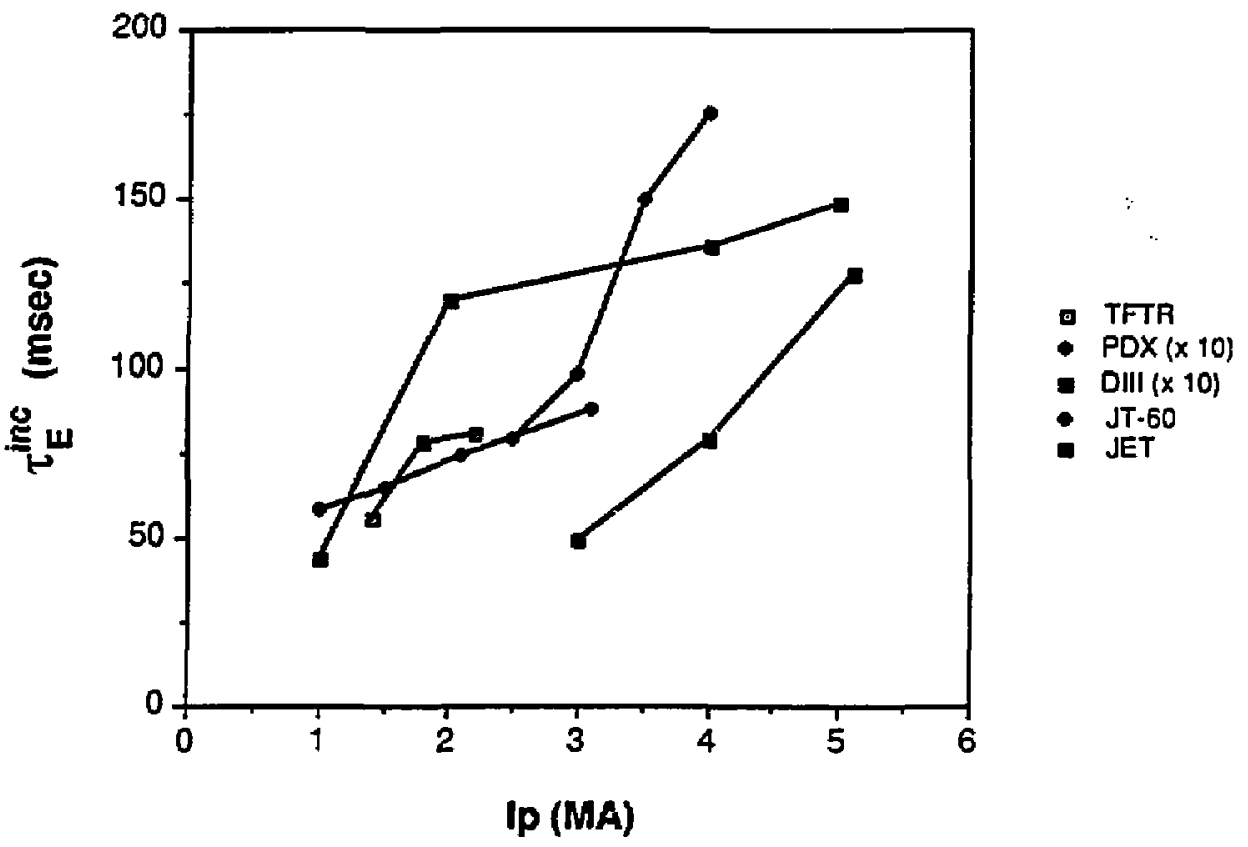

Figure 5 